\title{
Comparative Tumor Imaging and PDT Efficacy of HPPH Conjugated in the Mono- and Di-Forms to Various Polymethine Cyanine Dyes: Part - 2
}

\author{
Nadine S. James ${ }^{1}$, Tymish Y. Ohulchanskyy ${ }^{2}$, Yihui Chen ${ }^{1}$, Penny Joshi ${ }^{1}$, Xiang Zheng ${ }^{1}$, Lalit N. Goswami ${ }^{1}$, \\ and Ravindra K. Pandey ${ }^{\circledR}$ \\ 1. Photodynamic Therapy Center, Molecular and Cellular Biophysics and Biochemistry, Cell Stress Biology, Roswell Park Cancer Institute, \\ Buffalo, NY 14263; \\ 2. Institute for Lasers, Photonics and Biophotonics, University at Buffalo, Buffalo, NY 14221, USA.
}

$\square$ Corresponding author: Ravindra K. Pandey, Ph. D., PDT Center, Cell Stress Biology, Roswell Park Cancer Institute, Buffalo, NY 14263, Tel: 716-845-3203, Fax: 716-845-8920.

(c) Ivyspring International Publisher. This is an open-access article distributed under the terms of the Creative Commons License (http:/ / creativecommons.org/ licenses/by-nc-nd/3.0/). Reproduction is permitted for personal, noncommercial use, provided that the article is in whole, unmodified, and properly cited.

Received: 2013.01.21; Accepted: 2013.03.15; Published: 2013.08.21

\begin{abstract}
Previous reports from our laboratory have shown that a bifunctional agent obtained by conjugating a photosensitizer (HPPH) to a cyanine dye (CD) can be used for fluorescence image-guided treatment of tumor by photodynamic therapy (PDT). However, the resulting HPPH-CD conjugate showed a significant difference between the tumor-imaging and therapeutic doses. It was demonstrated that the singlet oxygen $\left({ }^{\prime} \mathrm{O}_{2}{ }^{*}\right.$, a key cytotoxic agent in PDT) produced by the conjugate upon excitation of the HPPH moiety was partially quenched by the CD-moiety; this resulted in a reduced PDT response when compared to HPPH-PDT under similar treatment parameters. To improve the therapeutic potential of the conjugate, we synthesized a series of dual functional agents in which one or two HPPH moieties were separately conjugated to three different dyes (Cypate, modified IR820 or modified IR783). The newly synthesized conjugates were compared with our lead compound HPPH-CD in terms of photophysical properties, in vitro and in vivo PDT efficacy, tumor uptake and imaging potential. Among the analogs investigated, the conjugate, in which two HPPH moieties were linked to the modified IR820 produced enhanced tumor uptake and tumor contrast in both Colon 26 (a murine Colon carcinoma) and U87 (a human glioblastoma) cell lines. The long-term PDT efficacy (cure) of this conjugate in BALB/c mice, bearing Colon 26 tumors was also enhanced; however, its efficacy in Nude mice bearing U87 tumors was slightly reduced. It was also found that in all the conjugates the singlet oxygen generation and, consequently, PDT efficacy were compromised by a competing pathway, whereby an electronic excitation of HPPH, the energy donor, is deactivated through an electronic excitation energy transfer (Forster Resonance Energy Transfer, FRET) to the CD fluorophore, the energy acceptor, resulting in overall reduction of the singlet oxygen production. Conjugates with increased FRET showed reduced singlet oxygen production and PDT efficacy. Among the conjugates investigated, the bifunctional agent in which two HPPH moieties were linked to the benzoindole-based cyanine dye II showed superiority over the lead candidate 9 (mono HPPH-cyanine dye).
\end{abstract}

Key words: Photodynamic therapy, Fluorophores, Reactive Oxygen species; Imaging. Forster Resonance Energy Transfer. 


\section{Introduction}

Photodynamic therapy (PDT) is a non-invasive treatment which combines three essential components: photosensitizer (PS), light of the appropriate wavelength, and oxygen to generate cytotoxic singlet oxygen $\left(\mathrm{CO}_{2}{ }^{*}\right)$. Immediately following light absorption, the PS is initially excited from its ground state (PS) to a short lived singlet state (1PS*) which converts to excited triplet state ( $\left.{ }^{3} \mathrm{PS}^{*}\right)$ through the process of intersystem crossing (ISC). Numerous studies have indicated that the dominant pathway in PDT is Type
II, whereby the ${ }^{3} \mathrm{PS}^{*}$ transfers energy to the molecular oxygen within tissue, resulting in the generation of reactive oxygen species (ROS), specifically, the oxygen molecule in the excited state, singlet oxygen $\left({ }^{1} \mathrm{O}_{2}{ }^{*}\right)$ [1-3]. Sufficient ${ }^{1} \mathrm{O}_{2}{ }^{*}$ generation leads to the irreversible destruction of diseased tissues without affecting surrounding healthy ones [3-5] (Figure 1). Systemically, PDT is also capable of the destruction of tumor tissue by invoking an immune response and vascular shutdown of the blood vessels surrounding the diseased tissue.

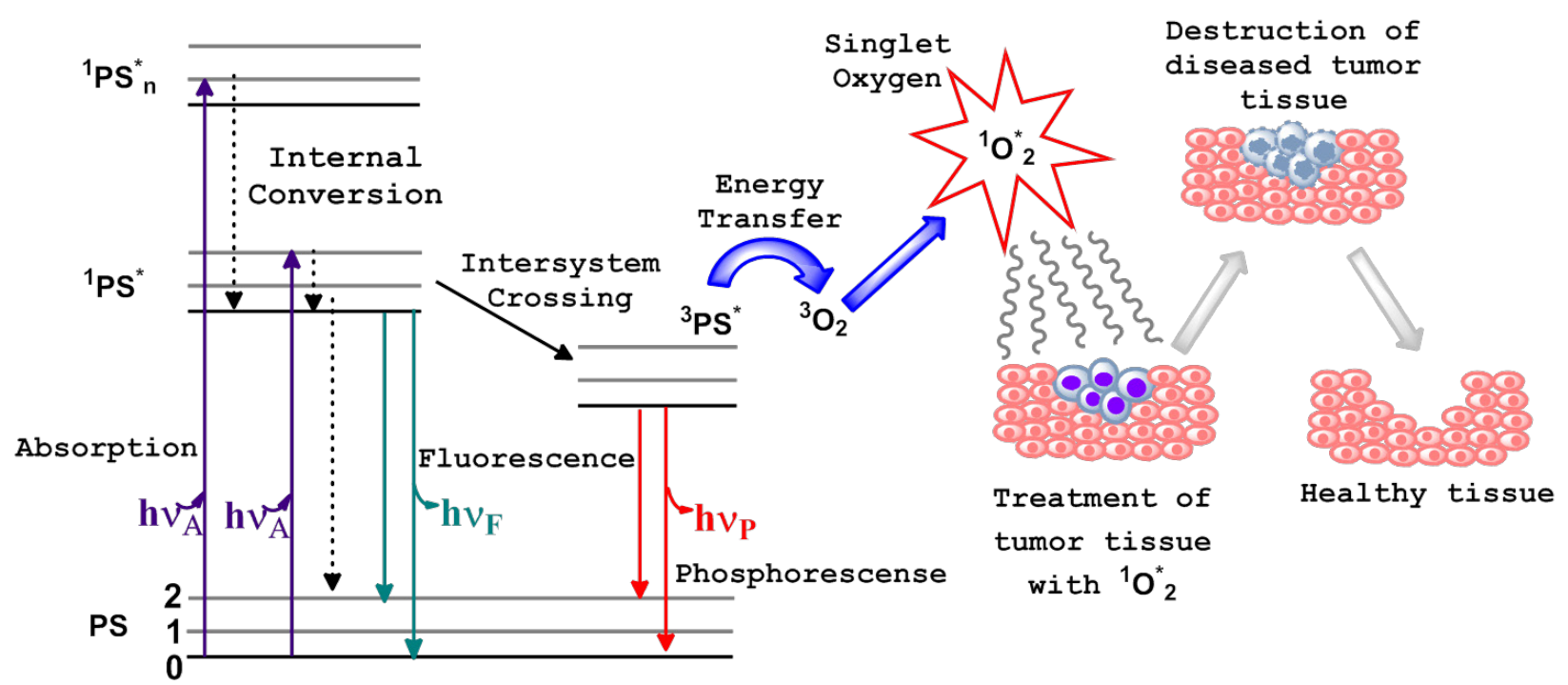

Fig I. Ablation of tumor cells after the three essential components (PS, light of the appropriate wavelength, and oxygen) of photodynamic therapy are combined. This usually results in the destruction of diseased tissue without affecting normal tissue. Jablonski diagram for the electronic states of the photosensitizer (PS) is shown on the left.

An important characteristic of most of the porphyrin-based photosensitizers is their ability to fluoresce. This property has been extensively explored in pre-clinical and clinical studies for fluorescence-image guided PDT. Unfortunately, the porphyrin or reduced porphyrin-based PS (chlorins, bacteriochlorins, and phthalocyanines) display small Stokes shift between their longer wavelength absorption and emission bands, and, therefore, their fluorescence is difficult to filter out of the scattered excitation light. This property of the PS fluorescence makes it not ideal for imaging deeply seated and large tumors. On the other hand, excitation of the photosensitizer pi-electron system ultimately leads to the production of the ${ }^{1} \mathrm{O}_{2}{ }^{*}$ and related toxicity. At the same time, presence of the fluorescent moiety within the structure of the PS-NIRF molecule, which can be independently and selectively excited with light of an appropriate wavelength, without the excitation of the PS pi-electron system, would allow for fluorescence imaging (optical tracking) of the PDT agent without toxic consequences.

Thus, using the PS-near infrared fluorophore (PS-NIRF) conjugate would be advantageous, because it can provide an opportunity to use a single molecule for both fluorescence imaging and PDT via the, so called, "see and treat approach" [6]. Near-infrared (NIR) polymethine-based cyanine dyes exhibit excellent photophysical properties with significant shifts between absorption and emission bands. Unfortunately, due to their limited tumor uptake and affinity, efforts are currently underway in our and other laboratories to modify them further by conjugating with a variety of tumor-targeted moieties. In a parallel study, we were interested in conjugating certain tumor-avid PS to the cyanine dyes (CD) and investigate their imaging and therapeutic potential.

We used this rationale in developing the $\mathrm{HPPH}-\mathrm{CD}$ conjugate. HPPH-CD was used as an efficient imaging agent for murine radiation-induced 
fibrosarcoma (RIF), Colon 26 [6], and human U87 glioblastoma $[7,8]$ at a dose of $0.3 \mu \mathrm{mol} / \mathrm{kg}$ or 0.03 $\mu \mathrm{mol} / \mathrm{kg}$. However, the therapeutic dose was found to be almost 10-fold to 100-fold higher than the imaging dose. We have confirmed that in the HPPH-CD conjugate, along with FRET between HPPH and CD moieties [9], another process takes place: a part of the singlet oxygen produced by irradiating the PS with light, reacts with the CD moiety, resulting in reduced singlet oxygen production by the conjugate. Therefore, we hypothesized that increasing the number of $\mathrm{HPPH}$ moieties in the conjugate should increase the overall singlet oxygen yield of the conjugate and long-term tumor cure by PDT.

\section{Materials and Methods}

\section{Chemistry}

2-[1'-Hexyloxyethyl]-2-devinyl pyropheophorbide-a (HPPH) was synthesized after many steps following the extraction of its precursor, crude chlorophyll-a, from Spirulina Pacifica Algae [10]. HPPH was linked to various aliphatic NBoc-protected amines via a peptide linkage. Upon de-protection these were conjugated to the commercially available NIRF (IR 820) upon modification with 3-mercaptobenzoic acid or 4-mercaptobenzoic in our laboratory. In addition, the NIRF cypate was synthesized using an already established procedure [11]. All reagents mentioned herein were purchased from Sigma Aldrich ${ }^{\circledR}$ and used without further purification. Photophysical experiments were carried out using spectroscopic grade solvents. The reactions were monitored by TLC and/or spectrophotometrically. Thin-layer chromatography (TLC) was done on ANALTECH pre-coated silica gel GF PE sheets (Cat. 159017, layer thickness $0.25 \mathrm{~mm}$ ). Purification was done by flash column chromatography performed over Silica Gel 60 (230 400 mesh). In some cases preparative TLC plates were also used for the purification (ANALTECH precoated silica gel GF glass plate, Cat. 02013, layer thickness 1.0 $\mathrm{mm})$. Dichloromethane was dried over $\mathrm{P}_{2} \mathrm{O}_{5}$ under $\mathrm{N}_{2}$ atmosphere. The synthetic intermediates and the final products were characterized by NMR $(400 \mathrm{MHz})$ and mass spectrometry (EIMS or HRMS). NMR spectra were recorded on a Varian $400 \mathrm{MHz}$ spectrometer at $303 \mathrm{~K}$ in $\mathrm{CDCl}_{3}$ or DMSO- $d_{6}$ solution and referenced to residual $\mathrm{CDCl}_{3}(7.26 \mathrm{ppm})$ or DMSO- $d_{6}(2.50 \mathrm{ppm})$. EI-Mass spectra were carried out on a Brucker Esquire ion-trap mass spectrometer equipped with a pneumatically assisted electrospray ionization source, operating in positive mode. UV-Visible spectrums were recorded on Varian Cary 50 Bio UV-Visible spectrophotometer using dichloromethane or methanol as solvent.

HPPH-NBoc-1, 2-ethylenediamine (HPPHNBoc-EDA). HPPH (6) (100 mg, 0.157 mmoles), N-ethyl-N'-(3-dimethylaminopropyl) carbodiimide hydrochloride (EDCI) (60 mg, 0.314 mmoles) and anhydrous dichloromethane (DCM) $(20 \mathrm{ml})$ were combined in a $50 \mathrm{ml}$ two neck rbf under Argon atm. Upon stirring the mixture for 30 minutes at room temperature (RT), 4-dimethylamino pyridine (DMAP) (38 mg, 0.314 mmoles) and NBoc-ethylenediamine (50 $\mathrm{mg}, 0.314$ mmoles) were added. This was stirred for 12 hours at RT. A crude TLC (eluted in a gradient of 5 - $20 \%$ acetone/DCM) of an aliquot of the mixture showed that the reaction was complete. Reaction was stopped by diluting with DCM and washing with brine. The aqueous layer (brine) was washed with DCM until it was clear. All organic layers were combined, dried over anhydrous sodium sulphate $\left(\mathrm{Na}_{2} \mathrm{SO}_{4}\right)$ and concentrated under vacuum. Purification was conducted via flash column chromatography using silica gel and eluted using the same gradient used to elute the crude TLC to obtain a black solid, (Pure yield $51 \mathrm{mg}, 42 \%$ ). UV-Vis $\lambda_{\max }$ (in $\mathrm{MeOH}$ ): 659.9 nm, 503 nm, $407.1 \mathrm{~nm} .{ }^{1} \mathrm{H}-\mathrm{NMR}$ (400 MHz, $\mathrm{CDCl}_{3}, \delta$ ppm) 9.76 (singlet, $1 \mathrm{H}$, meso-H), 9.21 (singlet, $1 \mathrm{H}$, meso-H), 8.52 (singlet, $1 \mathrm{H}$, meso- $\mathrm{H}$ ), 6.12 (brs, $1 \mathrm{H}, \mathrm{NH}), 5.92\left(\mathrm{~m}, 1 \mathrm{H}, \mathrm{CH}_{3} \mathrm{CH}_{\text {HOhexyl) }} 5.29\right.$ (d, $\left.1 \mathrm{H}, 15^{1}-\mathrm{CHH}, \mathrm{J}=19.6 \mathrm{~Hz}\right), 5.09\left(\mathrm{~d}, 1 \mathrm{H}, 15^{1}-\mathrm{CHH}, \mathrm{J}=\right.$ $20.0 \mathrm{~Hz}$ ), 4.85 (brs, 1H, NH), 4.52 (q, 1H, 17-H, J = 7.6 $\mathrm{Hz}), 4.30(\mathrm{~d}, 1 \mathrm{H}, \mathrm{H}-18, \mathrm{~J}=5.2 \mathrm{~Hz}), 3.62-3.61(\mathrm{~m}, 4 \mathrm{H}$, $8-\mathrm{CH}_{2} \mathrm{CH}_{3} \quad \& \quad-\mathrm{OCH}_{2}-\mathrm{Hexyl}$ ), 3.38 (singlet, $3 \mathrm{H}$, ring- $\mathrm{CH}_{3}$ ), 3.28 (singlet, $3 \mathrm{H}$, ring- $\mathrm{CH}_{3}$ ), 3.28 (singlet, $3 \mathrm{H}$, ring- $\left.\mathrm{CH}_{3}\right), 3.18\left(\mathrm{~m}, 2 \mathrm{H},-\left(\mathrm{NHCH}_{2}\right)_{2}-\right), 3.08(\mathrm{~m}, 2 \mathrm{H}$, $\left.-\left(\mathrm{NHCH}_{2}\right)_{2}\right)^{2}, 2.65\left(\mathrm{~m}, 1 \mathrm{H}, 17^{2}-\mathrm{CHH}\right), 2.45(\mathrm{~m}, 1 \mathrm{H}$, $\left.17^{2}-\mathrm{CH} \underline{\mathrm{H}}\right), 2.30\left(\mathrm{~m}, 1 \mathrm{H}, 171_{-} \mathrm{C} \overline{\mathrm{H}} \mathrm{H}\right), 2.13(\mathrm{~d}, 3 \mathrm{H}$, $\left.\mathrm{CH}_{3} \mathrm{CH}-O h e x y l, \mathrm{~J}=7.2 \mathrm{~Hz}\right), 2.05(\mathrm{~m}, 1 \mathrm{H}, 171-\mathrm{CHH})$, $1.80\left(\mathrm{~d}, 3 \mathrm{H}, 18-\mathrm{CH}_{3}, \mathrm{~J}=7.2 \mathrm{~Hz}\right), 1.75(\mathrm{~m}, 2 \mathrm{H}$, $\left.-\mathrm{CH}_{2}-\mathrm{Hexyl}\right), 1.63\left(\mathrm{t}, 3 \mathrm{H}, 8-\mathrm{CH}_{2} \mathrm{CH}_{3}, \mathrm{~J}=7.2 \mathrm{~Hz}\right), 1.43$ $\left(\mathrm{m}, 2 \mathrm{H},-\mathrm{CH}_{2}-\mathrm{Hexyl}\right), 1.24\left(\mathrm{~m}, 4 \mathrm{H},-2 \mathrm{CH}_{2}-\mathrm{Hexyl}\right), 1.21$ (s, 9H, NH-Boc), $0.80\left(\mathrm{t}, 3 \mathrm{H}, \mathrm{CH}_{3}-\mathrm{Hexyl}, \mathrm{J}=6.8 \mathrm{~Hz}\right.$ ), 0.45 (brs, $1 \mathrm{H}, \mathrm{NH}),-1.65$ (brs, $1 \mathrm{H}, \mathrm{NH}) ; \mathrm{m} / \mathrm{z}$ calculated for $[\mathrm{M}]^{+} \mathrm{C}_{46} \mathrm{H}_{62} \mathrm{~N}_{6} \mathrm{O}_{5}$ : 778.4782, found HRMS (TOFMS) $[\mathrm{MH}]^{+}$779.4851; low res (ESIMS) [MH] $]^{+}: 779.7$

HPPH-1,2-ethylenediamine (HPPH-EDA) (7). HPPH-NBoc-EDA (100 mg, 0.128 mmoles) was stirred with $50 \%$ TFA/ DCM $(5.0 \mathrm{ml})$ in a dry $50 \mathrm{ml} \mathrm{rbf}$ at RT for $3 \mathrm{hrs}$. Excess TFA was removed by concentrating the mixture under high vacuum. The resultant slush was diluted with DCM and washed with brine (x3) in order to remove traces of TFA. Organic fraction was collected, dried over $\mathrm{Na}_{2} \mathrm{SO}_{4}$ and concentrated under high vacuum to yield a black solid (Pure yield 50.5 $\mathrm{mg}, 58 \%$ ). UV-Vis $\lambda_{\max }$ (in MeOH): $660 \mathrm{~nm}, 605 \mathrm{~nm}$, 536 nm, 505 nm, 407.1 nm; ${ }^{1}$ H-NMR $\left(400 \mathrm{MHz}, \mathrm{CDCl}_{3}\right.$, 
$\delta$ ppm) 9.79 / 9.75 (singlet, 1H, meso-H), 8.98 (singlet, $1 \mathrm{H}$, meso-H), 8.47 (singlet, $1 \mathrm{H}$, meso- $\mathrm{H}), 5.94(\mathrm{~m}, 1 \mathrm{H}$, $\left.\mathrm{CH}_{3} \mathrm{C} \underline{\mathrm{H} O h e x y l}\right), 5.30(\mathrm{~d}, 1 \mathrm{H}, 151-\mathrm{C} \underline{\mathrm{H}}), 5.00(\mathrm{~d}, 1 \mathrm{H}$, $151-\mathrm{CH} \underline{\mathrm{H}}), 4.55(\mathrm{~m}, 1 \mathrm{H}, 17-\mathrm{H}, \mathrm{J}=7.6 \mathrm{~Hz}), 4.18(\mathrm{~m}, 1 \mathrm{H}$, $\mathrm{H}-18), 3.54-3.74\left(\mathrm{~m}, 4 \mathrm{H}, 8-\mathrm{CH}_{2} \mathrm{CH}_{3}\right.$ \& $\left.-\mathrm{OCH}_{2}-\mathrm{Hexyl}\right)$, $3.42,3.37,3.23$ (all singlet, $3 \mathrm{H}$ each, ring- $\left.\mathrm{CH}_{3}\right), 3.23(\mathrm{~m}$, $\left.2 \mathrm{H},-\left(\mathrm{NHCH}_{2}\right)_{2}-\right), 3.00\left(\mathrm{~m}, 2 \mathrm{H},-\left(\mathrm{NHCH}_{2}\right)_{2}\right), 2.65(\mathrm{~m}$, $\left.1 \mathrm{H}, 17^{2}-\mathrm{CHH}\right), 2.45\left(\mathrm{~m}, 1 \mathrm{H}, 17^{2}-\mathrm{CHH}\right), 2.20(\mathrm{~m}, 1 \mathrm{H}$, 171- $\mathrm{C} \underline{H}), 2.15$ (distorted $\mathrm{d}, 3 \mathrm{H}, \overline{\mathrm{C}}_{3} \mathrm{CH}-\mathrm{Ohexyl}$ ), $2.05\left(\mathrm{~m}, 1 \mathrm{H}, 171_{-} \mathrm{CHH}\right), 1.65-1.70(\mathrm{~m}, 8 \mathrm{H}, 2 \mathrm{H}$ for $-\mathrm{CH}_{2}-\mathrm{Hexyl}$ and $3 \mathrm{H}$ for $18-\mathrm{CH}_{3}, 3 \mathrm{H}$ for $\left.8-\mathrm{CH}_{2} \mathrm{CH}_{3}\right)$, $1.13-1.38\left(\mathrm{~m}, 6 \mathrm{H}, 3 \times-\mathrm{CH}_{2}-\mathrm{Hexyl}\right), 0.75(\mathrm{t}, 3 \mathrm{H}$, $\left.\mathrm{CH}_{3}-\mathrm{Hexyl}\right): \mathbf{m} / \mathbf{z}$ calculated for $[\mathrm{M}]^{+} \mathrm{C}_{41} \mathrm{H}_{54} \mathrm{~N}_{6} \mathrm{O}_{3}$ : 678.9059 , found low res (ESIMS) $[\mathrm{MH}]^{+}: 679.6$ found HRMS (TOFMS) $[\mathrm{MH}]^{+} \mathrm{C}_{41} \mathrm{H}_{55} \mathrm{~N}_{6} \mathrm{O}_{3}: 679.4346$

$\mathrm{HPPH}_{2}$-Diethylenetriamine (8): Diethylenetriamine (103.2 mg, $0.128 \mathrm{mmol})$, HPPH (6) $(162.2 \mathrm{mg}$, $0.255 \mathrm{mmol})$ and DMTMM $(81.2 \mathrm{mg}, 0.293 \mathrm{mmol})$ were all dissolved in dry DMF $(6 \mathrm{ml})$ and the solution was stirred under argon overnight. After the solvent was removed, the residue was purified by column chromatography using $\mathrm{MeOH} / \mathrm{DCM}(12 \%$ to $20 \%)$ as the elute solvent and the product (8) was obtained in $\sim 48 \%$ yield, the minor mono- product was obtained in a yield of $9 \%$. Both products were obtained as black solids. UV-VIS $\lambda_{\max }$ (in DCM), nm: $410\left(\varepsilon=20.0 \times 10^{4}\right)$, $505\left(\varepsilon=1.89 \times 10^{4}\right), 537\left(\varepsilon=1.9 \times 10^{4}\right), 605(\varepsilon=1.64 \times$ $\left.10^{4}\right), 660\left(\varepsilon=9.5 \times 10^{4}\right) .{ }^{1} \mathrm{H}$ NMR $(400 \mathrm{MHz}, \mathrm{CDCl} 3, \delta$ ppm): 9.72 (s, 1H, H-5), 9.71 (s, 1H, H-5), 8.83 (s, 1H, $\mathrm{H}-10), 8.75$ (s, 1H, H-10), 8.44 (s, 1H, H-20), 8.43 (s, 1H, H-20), 6.29 (br, 2H, 2x-CONH), 5.89 (m, 2H, H-31), 5.06 (m, 2H, H-18), 4.70 (m, 2H, H-17), 4.35 (m, 2H, H-132), 4.03 (m, 1H, H-132), 3.94 (m, 1H, H-132), 3.71 - 3.54 (m, $\left.4 \mathrm{H}, \quad 8-\mathrm{C}_{2} \mathrm{CH}_{3}\right), \quad 3.52-3.25 \quad(\mathrm{~m}, \quad 4 \mathrm{H}$, 31- $\left.\mathrm{OCH}_{2}\left(\mathrm{CH}_{2}\right)_{4} \mathrm{CH}_{3}\right), 3.35\left(\mathrm{~s}, 3 \mathrm{H}, 7-\mathrm{CH}_{3}\right), 3.33$ (s, $3 \mathrm{H}$, 7- $\left.\mathrm{CH}_{3}\right), 3.22\left(\mathrm{~s}, 3 \mathrm{H}, 2-\mathrm{CH}_{3}\right), 3.18\left(\mathrm{~s}, 3 \mathrm{H}, 2-\mathrm{CH}_{3}\right), 3.08$ $\left(\mathrm{m}, \quad 4 \mathrm{H}, \quad-\mathrm{CONHCH}_{2} \mathrm{CH}_{2} \mathrm{NHCH}_{2}\right.$ $\left.\mathrm{CH}_{2} \mathrm{NHCO}-\right), 2.93\left(\mathrm{~m}, 6 \mathrm{H}, 12-\mathrm{CH}_{3}\right), 2.45(\mathrm{~m}, 4 \mathrm{H}$, 17- $\left.\underline{\mathrm{H}}_{2} \mathrm{CH}_{2} \mathrm{CO}-\right), 2.25$ (m, 4H, 17- $\left.\mathrm{CH}_{2} \mathrm{CH}_{2} \mathrm{CO}-\right), 2.16$ $2.01\left(\mathrm{~m}, 4 \mathrm{H},-\mathrm{CONHCH}_{2} \mathrm{CH}_{2} \mathrm{NHCH}_{2} \mathrm{CH}_{2} \mathrm{NHCO}-\right)$, $2.10\left(\mathrm{~s}, 3 \mathrm{H}, 3-\mathrm{CHC}_{3}\right), 2.09\left(\mathrm{~s}, 3 \mathrm{H}, 3-\mathrm{CHC}_{3}\right), 2.00-$ $1.80\left(\mathrm{~m}, 4 \mathrm{H}, 3^{1}-\mathrm{OCH}_{2} \mathrm{CH}_{2}\left(\mathrm{CH}_{2}\right)_{3} \mathrm{CH}_{3}\right), 1.74(\mathrm{~d}, \mathrm{~J}=6.8$ $\left.\mathrm{Hz}, 6 \mathrm{H}, 18-\mathrm{CH}_{3}\right), 1.66\left(\mathrm{t}, \mathrm{J}=6.8 \mathrm{~Hz}, 6 \mathrm{H}, 8-\mathrm{CH}_{2} \mathrm{CH}_{3}\right), 1.47$ $\left(\mathrm{m}, 4 \mathrm{H}, 3{ }^{1}-\mathrm{OCH}_{2} \mathrm{CH}_{2} \mathrm{CH}_{2}\left(\mathrm{CH}_{2}\right)_{2} \mathrm{CH}_{3}\right), 1.26(\mathrm{~m}, 8 \mathrm{H}$, $\left.31-\mathrm{OCH}_{2} \mathrm{CH}_{2} \mathrm{CH}_{2}\left(\mathrm{CH}_{2}\right)_{2} \mathrm{CH}_{3}\right), 0.79(\mathrm{t}$, J $=7.3 \mathrm{~Hz}, 6 \mathrm{H}$, 31- $\left.\mathrm{OCH}_{2}\left(\mathrm{CH}_{2}\right)_{4} \mathrm{CH}_{3}\right), 0.29$ (br, $\left.2 \mathrm{H},-\mathrm{NH}-21\right),-1.68$ (s, $2 \mathrm{H},-\mathrm{NH}-23)$. HRMS for $\mathrm{C}_{82} \mathrm{H}_{105} \mathrm{~N}_{11} \mathrm{O}_{6}$, calculated for $\mathrm{MH}^{+}$: 1340.8327; found: 1340.8314 .

Synthesis of HPPH-CD (9): HPPH (100 mg) and $\mathrm{N}, \mathrm{N}^{\prime}$-dicyclohexylcarbodiimide (DCC) $(110 \mathrm{mg})$ were dissolved in $1 \mathrm{ml}$ dry DMF. After stirred for 10 minutes, the solution of NIRF 1 (60 mg) in DMF (2 ml) then $10 \mathrm{mg}$ DMAP were added. 24 hours later, the reaction mixture was worked up. The residue was purified by chromatography using $\mathrm{MeOH} / \mathrm{DCM}$ (1:3) as the elute solvent and 9 was obtained in $\sim 65 \%$ yield. UV-VIS in $\mathrm{H}_{2} \mathrm{O}: 848 \mathrm{~nm}\left(\varepsilon=9.8 \times 10^{4}\right), 664 \mathrm{~nm}(\varepsilon=5.4$ $\left.x 10^{4}\right), 413 \mathrm{~nm}\left(\varepsilon=10.2 \times 10^{4}\right)$. UV-VIS in MeOH: 833 $\mathrm{nm}\left(\varepsilon=20.7 \times 10^{4}\right), 660 \mathrm{~nm}\left(\varepsilon=5.4 \times 10^{4}\right), 408 \mathrm{~nm}(\varepsilon=$ $\left.9.5 \times 10^{4}\right)$. NMR $(\mathrm{CDCl} 3), \delta(\mathrm{ppm})$ for compound 9 : 9.47 (s, 1H, meso-H in HPPH part), 8.46 (s, 1H, meso-H in HPPH part), 8.35 (br-s, 3H, $1 \mathrm{H}$ for meso-H in HPPH part, $2 \mathrm{H}$ for $\mathrm{H}-\mathrm{a}), 7.50(\mathrm{~m}, 5 \mathrm{H}, 1 \mathrm{H}$ for $\mathrm{H}-\mathrm{b}, 4 \mathrm{H}$ for $\mathrm{H}-\mathrm{c})$, $7.30(\mathrm{~m}, 3 \mathrm{H}, 1 \mathrm{H}$ for $\mathrm{H}-\mathrm{b}, 2 \mathrm{H}$ for H-e), 7.20 (s, 2H, H-f), 7.05 (s, 4H, H-d), 6.85 (s, 2H, H-g), 6.61 (s, 2H, H-h), $5.70\left(\mathrm{br}, 3 \mathrm{H}, 1 \mathrm{H}\right.$ for $\mathrm{H}-{ }^{1}, 1 \mathrm{H}$ for $\mathrm{H}-17,1 \mathrm{H}$ for $\mathrm{H}-18$ ), 4.54 (br-doublet, 1H, H-132), 4.22 (br, 2H, H-i), 3.66 (br, $2 \mathrm{H}, \mathrm{H}-\mathrm{i}), 3.52\left(\mathrm{br}, 1 \mathrm{H}, \mathrm{H}-13^{2}\right), 3.20$ (br, 9H, $5 \mathrm{H}$ for $\mathrm{HPPH}$ part: $3 \mathrm{H}$ for $7-\mathrm{CH}_{3}, 2 \mathrm{H}$ for $3^{1}-\mathrm{OCH}_{2}\left(\mathrm{CH}_{2}\right)_{4} \mathrm{CH}_{3}, 4 \mathrm{H}$ for $\left.\mathrm{H}-\mathrm{j}\right), 3.03(\mathrm{~m}, 4 \mathrm{H}, \mathrm{H}-\mathrm{k})$, 2.90 (s, $1 \mathrm{H},-\mathrm{CON} \underline{\mathrm{H}}-), 2.72$ (br, $7 \mathrm{H}, 2 \mathrm{H}$ for $8-\mathrm{C}_{2} \mathrm{CH}_{3}$, $2 \mathrm{H}$ for $17-\mathrm{C}_{2} \mathrm{CH}_{2} \mathrm{CO}-, 3 \mathrm{H}$ for $\left.2-\mathrm{CH}_{3}\right), 2.55(\mathrm{br}, 5 \mathrm{H}, 2 \mathrm{H}$ for $17-\mathrm{CH}_{2} \underline{\mathrm{C}}_{2} \mathrm{CO}-, 3 \mathrm{H}$ for $\left.12-\overline{\mathrm{CH}}_{3}\right), 1.88(\mathrm{br}, 3 \mathrm{H}$, 3-CHC. ${ }_{3}$ ), 1.72-0.72 (many multiples, 36 protons, 22 $\mathrm{H}$ for dye part: $12 \mathrm{H}$ for $\mathrm{H}-\mathrm{p}, 4 \mathrm{H}$ for $\mathrm{H}-\mathrm{l}, 6 \mathrm{H}$ for $\mathrm{H}-\mathrm{m}$, n, o; $14 \mathrm{H}$ for $\mathrm{HPPH}$ part: $3 \mathrm{H}$ for $18-\mathrm{C}_{3}, 3 \mathrm{H}$ for $8-\mathrm{CH}_{2} \mathrm{CH}_{3}, 8 \mathrm{H}$ for $\left.3^{1}-\mathrm{OCH}_{2}\left(\mathrm{CH}_{2}\right)_{4} \mathrm{CH}_{3}\right), 0.62(\mathrm{~m}, 3 \mathrm{H}$, 31- $\mathrm{OCH}_{2}\left(\mathrm{CH}_{2}\right)_{4} \mathrm{C}_{3}$. MS calculated: $\mathrm{C}_{91} \mathrm{H}_{102} \mathrm{~N}_{7} \mathrm{~N}_{2} \mathrm{O}_{9} \mathrm{~S}_{3}$ : 1555.6, Found: 1555.7; HRMS calculated: $\mathrm{C}_{91} \mathrm{H}_{102} \mathrm{~N}_{7} \mathrm{~N}_{\mathrm{a}} \mathrm{O}_{9} \mathrm{~S}_{3}$ : 1555.6798, Found: $\mathrm{MH}^{+}$, $\mathrm{C}_{91} \mathrm{H}_{103} \mathrm{~N}_{7} \mathrm{~N}_{\mathrm{a}} \mathrm{O}_{9} \mathrm{~S}_{3} 1555.6920$.

Compound 10: HPPH $(76.4 \mathrm{mg}, 0.12 \mathrm{mmol})$, NIRF $2 \quad(100 \quad \mathrm{mg}, \quad 0.12 \quad \mathrm{mmol})$ and (4-(4,6-Dimethyoxy-1,3,5-triazin-2yl)-4-methylmorph olin-4-ium chloride) DMTMM $(36.5 \mathrm{mg}, 0.132 \mathrm{mmol})$ were all dissolved in dry DMF $(15 \mathrm{ml})$ and the solution was stirred under argon overnight. After the solvent was removed, the residue was purified by chromatography using a gradient of $(5-30 \%)$ $\mathrm{MeOH} / \mathrm{DCM}$ as the elute solvent and $\mathbf{1 0}$ was obtained in $\sim 60 \%$ yield as a black solid. ${ }^{1} \mathbf{H}$ NMR (400MHz, DMSO-d ${ }_{6} \delta$ ppm): 9.79 (s, $1 \mathrm{H}, \mathrm{H}-5$ of HPPH part), 9.75 (s, 1H, H-10 of HPPH part), 8.79 (s, 1H, $\mathrm{H}-20$ of HPPH part), $8.59(\mathrm{~m}, 2 \mathrm{H}$, aromatic protons of cyanine dye part), $7.38-7.48(\mathrm{~m}, 6 \mathrm{H}$, aromatic protons of cyanine dye part), $7.89-7.94(\mathrm{~m}, 6 \mathrm{H}$, aromatic protons of cyanine dye part), $7.33(\mathrm{t}, 2 \mathrm{H}), 7.13-7.17(\mathrm{~m}$, $4 \mathrm{H}), 6.33(\mathrm{~d}, 2 \mathrm{H}), 5.95-5.97\left(\mathrm{~m}, 1 \mathrm{H}, 3^{1}-\mathrm{H}\right.$ of $\mathrm{HPPH}$ part), 5.21 and $5.10\left(\mathrm{~d}, 2 \mathrm{H}, 13 \mathrm{CH}_{2}\right.$ of HPPH part), 4.54 - 4.56 and 4.30 - $4.33(\mathrm{~m}, 2 \mathrm{H}, 18-\mathrm{H}$ and $17-\mathrm{H}$ of $\mathrm{HPPH}$ part), $4.10-4.15\left(\mathrm{~m}, 4 \mathrm{H}, 2 \mathrm{X}-\mathrm{NCH}_{2}\left(\mathrm{CH}_{2}\right)_{3} \mathrm{SO}_{3}{ }^{-}\right), 3.69$ $\left(\mathrm{m}, 2 \mathrm{H}, 8-\mathrm{CH}_{2} \mathrm{CH}_{3}\right), 3.62\left(\mathrm{~s}, 3 \mathrm{H}, 7-\mathrm{CH}_{3}\right), 3.33-3.38$ (overlapped, $5 \mathrm{H}, 2 \mathrm{H}$ for ${ }^{3}-\mathrm{OCH}_{2}\left(\mathrm{CH}_{2}\right)_{4} \mathrm{CH}_{3}$ of $\mathrm{HPPH}$ part, and $3 \mathrm{H}$ of $\left.2-\mathrm{CH}_{3}\right), 3.20\left(\mathrm{~s}, 3 \mathrm{H}, 2 \mathrm{CH}_{3}\right), 2.70-2.75$ (m, $4 \mathrm{H}, 2 \mathrm{X}-\mathrm{N}\left(\mathrm{CH}_{2}\right)_{3} \mathrm{CH}_{2} \mathrm{SO}_{3}$ ), 2.45 (overlapped $\mathrm{m}, 6 \mathrm{H}$, $4 \mathrm{H}$ for $2 \mathrm{X}-\mathrm{NCH}_{2}\left(\mathrm{CH}_{2}\right) \mathrm{CH}_{2} \mathrm{CH}_{2} \mathrm{SO}_{3}$ - of cyanine dye part, $2 \mathrm{H}$ for $\left.17 \mathrm{CH}_{2} \mathrm{CH}_{2} \mathrm{COO}^{-}\right), 2.01-2.04(\mathrm{~m}, 3 \mathrm{H}$, $3^{2}-\mathrm{CH}_{3}$ of $\mathrm{HPPH}$ part), 1.90 (m, $\left.2 \mathrm{H}, 17 \mathrm{CH}_{2} \mathrm{CH}_{2} \mathrm{COO}^{-}\right)$, 
$1.60-1.75\left(\mathrm{~m}, 13 \mathrm{H}, 6 \mathrm{H}\right.$ for cyclohexene- $\left(\mathrm{CH}_{2}\right)_{3}{ }^{-}$of cyanine dye part, and $3 \mathrm{H}$ for $18-\mathrm{CH}_{3}$ and , $4 \mathrm{H}$ for $2 \mathrm{X}-\mathrm{NCH}_{2}\left(\mathrm{CH}_{2}\right) \mathrm{CH}_{2} \mathrm{CH}_{2} \mathrm{SO}_{3}{ }^{-}$of cyanine dye part), 1.55 (m, 3H for 8- $\mathrm{CH}_{2} \mathrm{CH}_{3}$ of HPPH part overlapped), 1.40 $1.42\left(14 \mathrm{H}, 12 \mathrm{H}\right.$ for $4 \mathrm{X}-\mathrm{CH}_{3}$ of cyanine dye part, $2 \mathrm{H}$ for $3^{1}-\mathrm{OCH}_{2} \mathrm{CH}_{2}\left(\mathrm{CH}_{2}\right)_{3} \mathrm{CH}_{3}$ of HPPH part), 1.25 - 1.22 (m, $\left.6 \mathrm{H}, 31-\mathrm{O}\left(\mathrm{CH}_{2}\right)_{2}\left(\mathrm{CH}_{2}\right)_{3} \mathrm{CH}_{3}\right), 0.62-0.66$ (m, 3H for 31- $\left.\mathrm{OCH}_{2}\left(\mathrm{CH}_{2}\right)_{4} \mathrm{CH}_{3}\right) ; \mathbf{m} / \mathbf{z}$ calculated for $[\mathrm{M}]^{+}$ $\mathrm{C}_{83} \mathrm{H}_{98} \mathrm{~N}_{7} \mathrm{NaO}_{9} \mathrm{~S}_{3}: 1456.89$, found low res (ESIMS) [M $\mathrm{Na}]^{-} \mathrm{C}_{83} \mathrm{H}_{98} \mathrm{~N}_{7} \mathrm{O}_{9} \mathrm{~S}_{3}: 1433.7$, found HRMS (TOFMS) [M - Na] ${ }^{-} \mathrm{C}_{83} \mathrm{H}_{98} \mathrm{~N}_{7} \mathrm{O}_{9} \mathrm{~S}_{3}: 1432.6560$

Compound 11: $\mathrm{HPPH}_{2}$ Diethylenetriamine 8 (134 $\mathrm{mg}, 0.1 \mathrm{mmol})$, NIRF 3 (100 mg, $0.1 \mathrm{mmol})$ and Benzotriazol-1-yloxytris(dimethyl-amino) phosphonium hexafluorophosphate (BOP) (54 mg, $0.122 \mathrm{mmol}$ ) were all dissolved in dry DMF (4 ml) and the solution was stirred under argon overnight. After the solvent was removed, the residue was purified by chromatography using $\mathrm{MeOH} / \mathrm{DCM}(\mathrm{v}: \mathrm{v}=1: 5)$ as the elute solvent and 11 was obtained in $\sim 60 \%$ yield $(137 \mathrm{mg})$. UV-VIS $\lambda_{\max }$ (in DCM), nm ( $\left.\varepsilon\right): 409\left(\varepsilon=17.4 \times 10^{4}\right), 506$ $\left(\varepsilon=2.2 \times 10^{4}\right), 539\left(\varepsilon=2.20 \times 10^{4}\right), 607\left(\varepsilon=1.86 \times 10^{4}\right)$, $661\left(\varepsilon=10.8 \times 10^{4}\right), 851\left(\varepsilon=19.0 \times 10^{4}\right) .{ }^{1} \mathbf{H}$ NMR $(400$ $\mathrm{MHz}, \mathrm{CDCl} 3, \delta \mathrm{ppm}): 9.57(\mathrm{~s}, 2 \mathrm{H}, 2 \mathrm{xH}-5$ of $\mathrm{HPPH}$ part), 8.73 (br, 2H, 2xH-10 of HPPH part), 8.36 (overlapped to be a singlet peak, $4 \mathrm{H}, 2 \mathrm{H}$ for $2 \times \mathrm{H}-20$ of $\mathrm{HPPH}$ part, $2 \mathrm{H}$ for two aromatic protons of cyanine dye part), 7.92 (br, $1 \mathrm{H}$, aromatic proton of cyanine dye part), 7.62 (overlapped to be a singlet peak, $3 \mathrm{H}$, aromatic protons of cyanine dye part), $7.11(\mathrm{~m}, 10 \mathrm{H}, 6 \mathrm{H}$ for aromatic protons of cyanine dye part, $2 \mathrm{H}$ for $-\mathrm{CH}=\mathrm{CH}-\mathrm{C}=\mathrm{C}-\mathrm{C}=\mathrm{CH}-\mathrm{CH}=\mathrm{C}-, 2 \mathrm{H}$ for aromatic protons of mercaptobenzoyl group), $6.73(\mathrm{~m}, 4 \mathrm{H}, 2 \mathrm{H}$ for $-\mathrm{CH}=\mathrm{CH}-\mathrm{C}=\mathrm{C}-\mathrm{C}=\mathrm{CH}-\mathrm{CH}=\mathrm{C}-, 2 \mathrm{H}$ for aromatic protons of mercaptobenzoyl group), $5.72(4 \mathrm{H}, 2 \mathrm{H}$ for $2 \times 3^{1}-\mathrm{H}, 2 \mathrm{H}$ for $\left.2 \mathrm{x}-\mathrm{CONH}-\right), 5.07(\mathrm{~m}, 2 \mathrm{H}, 2 \times 18-\mathrm{H}$ of HPPH part), 4.72 (m, 2H, 2x17-H of HPPH part), 4.33 $(\mathrm{m}, 2 \mathrm{H}, 132-\mathrm{H}$ of $\mathrm{HPPH}$ part), $3.84(\mathrm{~m}, 2 \mathrm{H}, 132-\mathrm{H}$ of $\mathrm{HPPH}$ part), $3.78-3.35 \quad(\mathrm{~m}, 18 \mathrm{H}, \quad 4 \mathrm{H}$ for $2 \mathrm{X}-\mathrm{NCH}_{2}\left(\mathrm{CH}_{2}\right)_{3} \mathrm{SO}_{3}{ }^{-}, 4 \mathrm{H}$ for $2 \mathrm{X}-\mathrm{N}\left(\mathrm{CH}_{2}\right)_{3} \mathrm{CH}_{2} \mathrm{SO}_{3}{ }^{-}, 6 \mathrm{H}$ for $2 \times 7-\mathrm{CH}_{3}$ of $\mathrm{HPPH}, 4 \mathrm{H}$ for $2 \times 3^{1}-\mathrm{OCH}_{2}\left(\mathrm{CH}_{2}\right)_{4} \mathrm{CH}_{3}$ of $\mathrm{HPPH}$ part), $3.35-2.77\left(\mathrm{~m}, 24 \mathrm{H}, 4 \mathrm{H}\right.$ for $2 \times 8-\mathrm{CH}_{2} \mathrm{CH}_{3}$, $4 \mathrm{H}$ for $2 \times 17-\mathrm{CH}_{2} \mathrm{CH}_{2} \mathrm{CO}-, 6 \mathrm{H}$ for $2 \times 2-\mathrm{CH}_{3}, 6 \mathrm{H}$ for $2 \times 12-\mathrm{CH}_{3}, 8 \mathrm{H}$ for $\left.-\mathrm{CONHCH}_{2} \mathrm{CH}_{2} \mathrm{NCH}_{2} \mathrm{CH}_{2} \mathrm{NHCO}-\right)$, $2.42\left(\mathrm{~m}, 4 \mathrm{H}, 2 \times 17-\mathrm{CH}_{2} \mathrm{CH}_{2} \mathrm{CO}-\right), 1.93(\mathrm{~m}, 12 \mathrm{H}, 6 \mathrm{H}$ for $2 \times 18-\mathrm{CH}_{3}, 6 \mathrm{H}$ for cyclohexene- $\left(\mathrm{CH}_{2}\right)_{3}$ - of cyanine dye part), $1.78-1.01\left(\mathrm{~m}, 48 \mathrm{H}, 12 \mathrm{H}\right.$ for $4 \mathrm{X}-\mathrm{CH}_{3}$ of cyanine dye part, $8 \mathrm{H}$ for $2 \mathrm{X}-\mathrm{NCH}_{2}\left(\mathrm{CH}_{2}\right)_{2} \mathrm{CH}_{2} \mathrm{SO}_{3}{ }^{-}$of cyanine dye part, $16 \mathrm{H}$ for $2 \times 3^{1}-\mathrm{OCH}_{2}\left(\underline{\mathrm{CH}}_{2}\right)_{4} \mathrm{CH}_{3}$ of $\mathrm{HPPH}$ part, $6 \mathrm{H}$ for $2 \times 8-\mathrm{CH}_{2} \mathrm{CH}_{3}$ of $\mathrm{HPPH}$ part, $6 \mathrm{H}$ for $2 \times 18-\mathrm{CH}_{3}$ of HPPH part), 0.71 (two triplet overlapped to be quartet, $\left.6 \mathrm{H}, \mathrm{J}=6.5 \mathrm{~Hz}, 3^{1}-\mathrm{OCH}_{2}\left(\mathrm{CH}_{2}\right)_{4} \mathrm{CH}_{3}\right), 0.08$ (br, 21-H of HPPH part), -1.89 (s, 23-H of HPPH part). HRMS for $\mathrm{C}_{135} \mathrm{H}_{159} \mathrm{~N}_{13} \mathrm{O}_{13} \mathrm{~S}_{3}$, calculated for $[\mathrm{M}-\mathrm{Na}] \mathrm{H}^{+}$:
2266.1342; found: 2266.1423 (double charge method).

Compound 12: $\quad \mathrm{HPPH}_{2}$ Diethylenetriamine 8 (82.5 mg, $0.0616 \mathrm{mmol})$, NIRF 4 (53.4 mg, 0.0616 mmol) and DMTMM (22.2 mg, $0.08 \mathrm{mmol})$ were all dissolved in dry DMF (15 ml) and the solution was stirred under argon overnight. After the solvent was removed, the residue was purified by chromatography using a gradient of $(5-30 \%) \mathrm{MeOH} / \mathrm{DCM}$ as the elute solvent and 12 was obtained in $\sim 60 \%$ yield as a black solid. ${ }^{1} \mathrm{H}$ NMR $\left(400 \mathrm{MHz}, \mathrm{DMSO}-\mathrm{d}_{6} \delta \mathrm{ppm}\right)$ : $9.60(\mathrm{~s}, 2 \mathrm{H}), 9.55(\mathrm{~s}, 1 \mathrm{H}), 9.52(\mathrm{~s}, 1 \mathrm{H}), 8.59(\mathrm{~s}, 1 \mathrm{H}), 8.57$ $(\mathrm{s}, 1 \mathrm{H}), 8.79(\mathrm{~s}, 1 \mathrm{H}, \mathrm{H}-20$ of HPPH part), 7.77-7.79 (m, $4 \mathrm{H}$, aromatic protons of cyanine dye part), $7.57-7.64$ $(\mathrm{m}, 2 \mathrm{H}$, aromatic protons of cyanine dye part), 6.80-7.10 (m, 4H) , 6.70-6.73 (m, 4H), 5.73-5.90 (m, 4H, $2 \mathrm{H}$ for cyanine dye part, $2 \mathrm{H}$ for $3^{1}-\mathrm{H}$ of $\mathrm{HPPH}$ part), $4.69-4.90\left(\mathrm{~m}, 8 \mathrm{H}, 4 \mathrm{H}\right.$ for $13 \mathrm{CH}_{2}$ of $\mathrm{HPPH}$ part, and $2 \mathrm{H}$ each for 18-H and 17-H of HPPH part), 4.02-4.05 (m, $\left.4 \mathrm{H}, 2 \mathrm{X}-\mathrm{NCH}_{2}\left(\mathrm{CH}_{2}\right)_{3} \mathrm{SO}_{3}{ }^{-}\right), 3.55\left(\mathrm{~m}, 4 \mathrm{H}, 8-\mathrm{CH}_{2} \mathrm{CH}_{3}\right)$, $3.40\left(\mathrm{~m}, 4 \mathrm{H}, 3^{1}-\mathrm{OCH}_{2}\left(\mathrm{CH}_{2}\right)_{4} \mathrm{CH}_{3}\right.$ of $\mathrm{HPPH}$ part), 3.10 -3.40 (overlapped s, $12 \mathrm{H}, 7-\mathrm{CH}_{3}$ and $2-\mathrm{CH}_{3}$ ), 3.08/3.10 $\left(\mathrm{s}, 6 \mathrm{H}, 12 \mathrm{CH}_{3}\right), 2.80\left(\mathrm{~m}, 4 \mathrm{H}, 2 \mathrm{X}-\mathrm{N}\left(\mathrm{CH}_{2}\right)_{3} \mathrm{CH}_{2} \mathrm{SO}_{3}\right), 2.49$ ( $2 \mathrm{H}$ for $17 \mathrm{CH}_{2} \mathrm{CH}_{2} \mathrm{COO}$ ), 2.45 (overlapped $\mathrm{m}, 4 \mathrm{H}$ for $2 \mathrm{X}-\mathrm{NCH}_{2}\left(\mathrm{CH}_{2}\right) \mathrm{CH}_{2} \mathrm{CH}_{2} \mathrm{SO}_{3}$ - of cyanine dye part), 2.35 $\left(\mathrm{m}, 6 \mathrm{H}, 3^{2}-\mathrm{CH}_{3}\right.$ of $\mathrm{HPPH}$ part $), 2.25(\mathrm{~m}, 2 \mathrm{H}$, $\left.17 \mathrm{CH}_{2} \mathrm{CH}_{2} \mathrm{COO}\right), 1.70-1.90(\mathrm{~m}, 16 \mathrm{H}, 6 \mathrm{H}$ for cyclohexene- $\left(\mathrm{CH}_{2}\right)_{3}$ - of cyanine dye part, and $6 \mathrm{H}$ for 18 $\mathrm{CH}_{3}$ and , $4 \mathrm{H}$ for $2 \mathrm{X}-\mathrm{NCH}_{2}\left(\mathrm{CH}_{2}\right) \mathrm{CH}_{2} \mathrm{CH}_{2} \mathrm{SO}_{3}{ }^{-}$of cyanine dye part), 1.45 (overlapped $22 \mathrm{H}, 12 \mathrm{H}$ for $4 \mathrm{X}-\mathrm{CH}_{3}$ of cyanine dye part, $4 \mathrm{H}$ for $3^{1}-\mathrm{OCH}_{2} \mathrm{CH}_{2}\left(\mathrm{CH}_{2}\right)_{3} \mathrm{CH}_{3}$ of $\mathrm{HPPH}$ part, $3 \mathrm{H}$ for $\left.8-\mathrm{CH}_{2} \mathrm{CH}_{3}\right), 1.15-1.29(\mathrm{~m}, 12 \mathrm{H}$, $31-\mathrm{O}\left(\mathrm{CH}_{2}\right)_{2}\left(\mathrm{CH}_{2}\right)_{3}$

$\left.\mathrm{CH}_{3}\right), 0.49-0.58\left(\mathrm{~m}, 3 \mathrm{H}\right.$ for $\left.3^{1}-\mathrm{OCH}_{2}\left(\mathrm{CH}_{2}\right)_{4} \mathrm{CH}_{3}\right) . \mathbf{m} / \mathbf{z}$ calculated for $[\mathrm{M}+\mathrm{Na}]^{+} \mathrm{C}_{127} \mathrm{H}_{154} \mathrm{~N}_{13} \mathrm{NaO}_{13} \mathrm{~S}_{3}: 2189.85$, found low res (ESIMS) [M+ Na] $]^{+}: 2189$, found HRMS (TOFMS) $[\mathrm{M}+\mathrm{Na}]^{+} \mathrm{C}_{127} \mathrm{H}_{154} \mathrm{~N}_{13} \mathrm{NaO}_{13} \mathrm{~S}_{3}: 2190.8993$.

Mono- and di-HPPH Cypate Conjugates (13, 14): HPPH-N-Boc ethylenediamine $(100.0 \mathrm{mg}, 0.128$ mmol) was taken in a dry RBF $(50.0 \mathrm{ml})$ and stirred with 50\% TFA/DCM (5.0 ml) at RT for $3 \mathrm{hr}$. Resultant mixture was concentrated under high vacuum. The remaining film washed with di-ionized water $(x 3)$ and DCM to remove excess TFA, organic layer was separated, dried over anhydrous $\mathrm{Na}_{2} \mathrm{SO}_{4}$ then concentrated under high vacuum. The crude thus obtained was dissolved in anhydrous DMF $(25 \mathrm{ml})$ and cypate (5) (32.0 $\mathrm{mg}, 0.051 \mathrm{mmol})$, $N$-Ethyl- $N$ '-(3-dimethylaminopropyl) carbodiimide hydrochloride (49.2 $\mathrm{mg}, \quad 0.257 \mathrm{mmol})$ and N-hydroxybenzotriazole $(34.7 \mathrm{mg}, 0.257 \mathrm{mmol}$ ) were added and the resultant mixture was stirred for $12 \mathrm{hr}$ at room temperature under $\mathrm{N}_{2}$ atmosphere. Reaction mixture was then diluted with dichloromethane (50.0 $\mathrm{ml})$ and washed with brine $(50 \mathrm{ml})$. Organic layer 
separated, dried over sodium sulfate and concentrated. Product was purified over silica gel column using 5-20\% methanol-dichloromethane as mobile phase. Yield of mono-conjugate (13): $10 \mathrm{mg}(16.5 \%)$. UV-Vis $\lambda_{\max }\left(\right.$ in $\left.\mathrm{CH}_{2} \mathrm{Cl}_{2}\right): 814 \mathrm{~nm}\left(\varepsilon 6.5 \times 10^{4}\right), 661 \mathrm{~nm}(\varepsilon$ $\left.3.6 \times 10^{4}\right), 608 \mathrm{~nm}\left(\varepsilon 0.7 \times 10^{4}\right), 538 \mathrm{~nm}\left(\varepsilon 0.6 \times 10^{4}\right), 506$ $\mathrm{nm}\left(\varepsilon 0.6 \times 10^{4}\right)$, and $412 \mathrm{~nm}\left(\varepsilon 5.6 \times 10^{4}\right) .{ }^{1} \mathrm{HNMR}$ (400Mhz, $\mathrm{CDCl}_{3}$ ): $\delta$ 9.62/9.60 (splitted singlet, $2 \mathrm{H}$, meso-H), 9.28 (splitted singlet, $2 \mathrm{H}$, meso-H), 8.44 (singlet, 2H, meso-H), $8.0(\mathrm{~m}, 2 \mathrm{H}$, Cypate- $\mathrm{CH}), 7.90$ $(\mathrm{m}, 2 \mathrm{H}$, Cypate- $\mathrm{CH}), 7.80(\mathrm{~m}, 2 \mathrm{H}$, Cypate- $\mathrm{CH}), 7.66$ $(\mathrm{m}, 4 \mathrm{H}$, Cypate- $\mathrm{CH}), 7.50(\mathrm{~m}, 2 \mathrm{H}$, Cypate- $\mathrm{CH}), 7.45$ $(\mathrm{m}, 2 \mathrm{H}$, Cypate- $\mathrm{CH}), 7.32(\mathrm{~m}, 2 \mathrm{H}$, Cypate-CH), 7.20 (m, 2H, Cypate- $\mathrm{CH}), 6.14-6.17$ (m, 1H, Cypate- $\mathrm{CH})$, 5.76-5.81 (m, 2H, $\left.\mathrm{CH}_{3} \mathrm{CHOhexyl}\right), 5.21 \& 4.97(\mathrm{~m}, 2 \mathrm{H}$, 131- $\left.\mathrm{CH}_{2}\right), 4.46-4.48(\mathrm{~m}, 2 \mathrm{H}, 17-\mathrm{H}), 4.06-4.19(5 \mathrm{H}, 1 \mathrm{H}$ of $18 \mathrm{H}$ and $4 \mathrm{H}$ of $2 \mathrm{CH}_{2}$ cypate), 3.51-3.57 $(\mathrm{m}, 4 \mathrm{H}$, 8- $\mathrm{CH}_{2} \mathrm{CH}_{3} \quad \& \quad-\mathrm{OCH}_{2}-\mathrm{Hexyl}$ ), 3.49 (singlet, $3 \mathrm{H}$, ring- $\mathrm{CH}_{3}$ ), 3.32 (singlet, $3 \mathrm{H}$, ring- $\left.\mathrm{CH}_{3}\right), 3.25-3.31(\mathrm{~m}$, $4 \mathrm{H}, 2 \mathrm{CH}_{2}-\mathrm{Cypate}$ ), 3.14 (singlet, $3 \mathrm{H}$, ring- $\mathrm{CH}_{3}$ ), 2.50-2.62 (m, 4H, $\left.\left.-\left(\mathrm{NHCH}_{2}\right)_{2}\right)^{-}\right), 2.41-2.49(\mathrm{~m}, 2 \mathrm{H}$, $\left.17^{2}-\mathrm{CH}_{2}\right), 2.25-2.30\left(\mathrm{~m}, 1 \mathrm{H}, 17^{1}-\mathrm{CH} \underline{\mathrm{H}}\right), 2.20-2.25(\mathrm{~m}$, $1 \mathrm{H}, 171-\mathrm{CH}), 1.92-2.00\left(\mathrm{~m}, 3 \mathrm{H}, \mathrm{C}_{3} \mathrm{CH}-O h e x y\right)$, 1.60-1.75 (broad, $16 \mathrm{H}, 12 \mathrm{H}$ of $\mathrm{CH}_{3}$-Cypate, $4 \mathrm{H}$ of- $\left.\mathrm{CH}_{2}-\mathrm{Hexyl}\right), 1.59\left(\mathrm{~m}, 3 \mathrm{H}, 18-\mathrm{CH}_{3}\right), 1.10-1.25(\mathrm{~m}, 5 \mathrm{H}$, 8- $\mathrm{CH}_{2} \mathrm{CH}_{3}$ and $\left.2 \mathrm{CH}_{2}-\mathrm{Hexyl}\right), 0.64-0.69(\mathrm{~m}, 3 \mathrm{H}$, $\mathrm{CH}_{3}$-Hexyl). Yield of di-conjugate (14): $45.0 \mathrm{mg}$ (49\%). UV-Vis $\lambda_{\max }\left(\right.$ in $\left.\mathrm{CH}_{2} \mathrm{Cl}_{2}\right): 806 \mathrm{~nm}\left(\varepsilon 14.4 \times 10^{4}\right), 661 \mathrm{~nm}$ $\left(\varepsilon 7.9 \times 10^{4}\right), 606 \mathrm{~nm}\left(\varepsilon 1.4 \times 10^{4}\right), 537 \mathrm{~nm}\left(\varepsilon 1.3 \times 10^{4}\right)$, $505 \mathrm{~nm}\left(\varepsilon 1.3 \times 10^{4}\right)$, and $411 \mathrm{~nm}\left(\varepsilon 14.1 \times 10^{4}\right) .{ }^{1} \mathrm{HNMR}$ $\left(400 \mathrm{Mhz}, \mathrm{CDCl}_{3}\right)$ : $\delta 9.69$ (splitted singlet, $2 \mathrm{H}$, meso-H), 9.15 (splitted singlet, 2H, meso-H), 8.47 (singlet, 2H, meso-H), 8.11 (m, 2H, Cypate- $\mathrm{CH}), 7.70(\mathrm{~m}, 2 \mathrm{H}$, Cypate-CH), $7.71(\mathrm{~m}, 2 \mathrm{H}$, Cypate- $\mathrm{CH}), 7.66(\mathrm{~m}, 4 \mathrm{H}$, Cypate-CH), 7.49 (m, 2H, Cypate-CH), $7.40(\mathrm{~m}, 2 \mathrm{H}$, Cypate- $\mathrm{CH}), 7.32(\mathrm{~m}, 2 \mathrm{H}, \mathrm{Cypate}-\mathrm{CH}), 7.20(\mathrm{~m}, 2 \mathrm{H}$, Cypate-CH), $6.42(\mathrm{~m}, 2 \mathrm{H}, \mathrm{NH}), 5.96(\mathrm{~m}, 1 \mathrm{H}, \mathrm{Cy}-$ pate- $\mathrm{CH})$, 5.85-5.79 (m, 2H, $\left.\mathrm{CH}_{3} \mathrm{CHOhexyl}\right)$, 5.24-5.17 (m, 2H, 151-CHH) $4.97-4.89\left(\mathrm{~m}, \overline{2 \mathrm{H}}, 15^{1}-\mathrm{CHH}\right), 4.50$ $(\mathrm{m}, 2 \mathrm{H}, 17-\mathrm{H}), 4.13(\mathrm{~m}, 2 \mathrm{H}, \mathrm{H}-18), 4.05-3.96(\mathrm{~m}, 4 \mathrm{H}$, $2 \mathrm{CH}_{2}$-Cypate), $3.61-3.48\left(\mathrm{~m}, 8 \mathrm{H}, 8-\mathrm{CH}_{2} \mathrm{CH}_{3} \quad \&\right.$ $-\mathrm{OCH}_{2}-\mathrm{Hexyl}$ ), 3.34 (splitted singlet, $6 \mathrm{H}$, ring- $\mathrm{CH}_{3}$ ), 3.31 (splitted singlet, $6 \mathrm{H}$, ring- $\left.\mathrm{CH}_{3}\right), 3.25-3.23(\mathrm{~m}, 4 \mathrm{H}$, $2 \mathrm{CH}_{2}$-Cypate), 3.19 (splitted singlet, $6 \mathrm{H}$, ring- $\mathrm{CH}_{3}$ ), $3.15\left(\mathrm{~m}, 4 \mathrm{H}, \quad-\left(\mathrm{NHCH}_{2}\right)_{2}\right), \quad 2.67-2.62(\mathrm{~m}, 4 \mathrm{H}$, $\left.-\left(\mathrm{NHCH}_{2}\right)_{2-}\right), 2.60-2.47\left(\mathrm{~m}, 4 \mathrm{H}, 1{ }^{2}-\mathrm{CH}_{2}\right), 2.32-2.26(\mathrm{~m}$, $2 \mathrm{H}, 171-\mathrm{CH} \underline{\mathrm{H}}), 2.20-2.14(\mathrm{~m}, 2 \mathrm{H}, 171-\mathrm{CHH}), 2.03(\mathrm{~m}$, $6 \mathrm{H}, \mathrm{CH}_{3} \mathrm{CH}$-Ohexyl), 1.67 (singlet, $12 \mathrm{H}, \mathrm{CH}_{3}-\mathrm{Cypate)}$, $1.62\left(\mathrm{~m}, 4 \mathrm{H},-\mathrm{CH}_{2}-\mathrm{Hexyl}\right), 1.55\left(\mathrm{~m}, 6 \mathrm{H}, 18-\mathrm{CH}_{3}\right)$, 1.43-1.23 (m, 12H, 6CH - - Hexyl), $1.19(\mathrm{~m}, 6 \mathrm{H}$, 8- $\left.\mathrm{CH}_{2} \mathrm{CH}_{3}\right), 0.78-0.72\left(\mathrm{~m}, 6 \mathrm{H}, \mathrm{CH}_{3}-\mathrm{Hexyl}\right), 0.37$ (brs, $2 \mathrm{H}, \mathrm{NH}),-1.72$ (brs, $2 \mathrm{H}, \mathrm{NH}) . \mathbf{m} / \mathbf{z}$ calculated for $[\mathrm{M}]^{+}$ $\mathrm{C}_{82} \mathrm{H}_{93} \mathrm{~N}_{8} \mathrm{O}_{6}+$ : 1286.67, $\mathrm{C}_{123} \mathrm{H}_{145} \mathrm{~N}_{14} \mathrm{O}_{8}+$ : 1947.56, found low res (ESIMS) $[\mathrm{M}]^{+} \quad \mathrm{C}_{82} \mathrm{H}_{93} \mathrm{~N}_{8} \mathrm{O}_{6}+:$ 1286.67;
$\mathrm{C}_{123} \mathrm{H}_{145} \mathrm{~N}_{14} \mathrm{O}_{8}+$ : 1947.6 found HRMS (TOFMS) [M] ${ }^{+}$ $\mathrm{C}_{82} \mathrm{H}_{93} \mathrm{~N}_{8} \mathrm{O}_{6}+: \quad 1285.7411 ; \quad[\mathrm{M}]^{+} \quad \mathrm{C}_{123} \mathrm{H}_{145} \mathrm{~N}_{14} \mathrm{O}_{8}+$ : 1946.1394.

In vitro studies: In vitro tumor cell uptake, cell phototoxicity assay and intracellular localization were performed in Colon 26, and/or U87 cell lines.

In vivo studies: In vivo tumor uptake, in vivo PDT efficacy and in vivo imaging were performed in BALB/c mice inoculated with Colon 26, and/or nude mice bearing U87 tumors.

Formulation: The compounds were formulated in $1 \%$ Tween-80 in D5W.

Photophysical characterization: UV-VIS absorption spectra of compounds (MeOH solutions) were acquired using a Shimadzu UV-3600 spectrophotometer. Fluorescence spectra were recorded using a Fluorolog-3 spectrofluorometer or a SPEX 270M Spectrometer (Jobin Yvon, Longjumeau, France). The SPEX 270M Spectrometer was utilized for measurements in NIR range; laser lines from Argon ion laser (Spectra Physics) or laser diodes emitting at 630 and $785 \mathrm{~nm}$ was used as excitation. Singlet oxygen, ${ }^{1} \mathrm{O}_{2}{ }^{*}$, generation was detected by its phosphorescence emission signal at $1270 \mathrm{~nm}$. A SPEX 270M Spectrometer equipped with Hamamatsu IR-PMT was used for recording singlet oxygen phosphorescence spectra. The sample placed in a quartz cuvette was positioned directly in front of the entrance slit of the spectrophotometer, and the emission signal was collected at $90^{\circ}$ relative to the excitation laser beam. Additional long-pass filters [a 950 LP filter and a 538 AELP filter (both from Omega Optical)] were used to attenuate the scattered light and fluorescence from the samples. ${ }^{1} \mathrm{O}_{2}{ }^{*}$ phosphorescence decays at $1270 \mathrm{~nm}$ was acquired using Infinium oscilloscope (Hewlett-Packard) coupled to the output of the PMT. A second harmonic (532 nm) from a nanosecond pulsed Nd: YAG laser (Lotis TII, Belarus) operating at $20 \mathrm{~Hz}$ was used as the excitation source in this case.

In vitro tumor models: Colon 26 cells were grown in sterile RPMI-1640, 1x with L-glutamine with $10 \%$ Fetal Calf Serum (FCS) (Atlanta Biologicals, triple $0.1 \mu \mathrm{m}$ filtered, Lawrenceville, GA), and $1 \%$ Penicillin/Steptomycin/L-glutamine (P/S/l-G 10,000 I.U/ml penicillin, 10,000 $\mu \mathrm{g} / \mathrm{ml}$ streptomycin, 29.2 $\mathrm{mg} / \mathrm{ml}$ L-glutamine) was maintained in $5 \% \mathrm{CO}_{2}, 95$ $\%$ air and $100 \%$ humidity. U87, gliobastoma astrocytoma, cells were grown in Medium Essential Medium Eagle (MEM), 1x with Earle's Salt and L-glutamine, sterile with $10 \%$ FCS, $1 \%$ P/S/l-G, $1 \%$ MEM Non-essential Amino Acids 100x solution, sterile, $1 \%$ Sodium Pyruvate, $100 \mathrm{mM}$ solution, sterile, and $1 \%$ $\mathrm{P} / \mathrm{S} / \mathrm{l}-\mathrm{G}$ and maintained in $5 \% \mathrm{CO}_{2}, 95 \%$ air and 100 $\%$ humidity. All reagents, except FCS, but including 
Trypsin/EDTA, 1x (0.25 \% Trypsin / 2.21 mM EDTA in HBSS without sodium bicarbonate, calcium and magnesium, sterile, Porcine Parvovirus tested) and DPBS, 1x (Dulbecco's Phosphate Buffered Saline), without calcium and magnesium, sterile were purchased from MediaTech, Inc., Manassas VA 20109. The 96 and 6 well plates were purchased from VWR. The MTT (3-[4,5-dimethylthiazol-2-yl]-2,5diphenyltetrazolium bromide) cell viability assays were read on a microtiter plate reader at an absorbance of $560 \mathrm{~nm}$. All compounds were formulated in 1 $\%$ Tween-80/D5W for solubility in aqueous solution and diluted in complete medium for all in vitro studies.

Determination of cell viability: Colon 26 and U87 cells were incubated in 96-well plates with the PS-NIRF conjugates at various drug concentrations ranging from $0-4 \mu \mathrm{M}$ for $24 \mathrm{~h}$. Upon irradiation the cells were incubated $44 \mathrm{~h}$ at $37^{\circ} \mathrm{C}$ in the dark. The cells were later treated with $10 \mu \mathrm{l}$ of $4.0 \mathrm{mg} / \mathrm{ml}$ solution of MTT dissolved in PBS. This was allowed to incubate for another $4 \mathrm{~h}$. After $4 \mathrm{~h}$ the MTT was removed and $100 \mu$ of dimethyl sulfoxide was added to solubilize the formazan crystals. The PDT efficacy was measured by reading the 96-well plate on a microtiter plate reader at an absorbance of $560 \mathrm{~nm}$. The results were plotted with the corresponding drug dose at each fluence $\left(\mathrm{J} / \mathrm{cm}^{2}\right)$ using the program OriginPro 8.6. The $\mathrm{EC}_{50}$ doses were calculated for each compound at the drug concentration of $4 \mu \mathrm{M}$ to compare their efficacy.

Animal and tumor models: Prior to commencement of in vivo studies all procedures or protocols were approved by the institutional animal care committee (IACUC). BALB/c mice 5-8 weeks of age were obtained from NCI Jackson Laboratory. The mice were inoculated s.c. on the right posterior shoulder with Colon 26 ( $1 \times 10^{6}$ cells in $50 \mu \mathrm{l}$ medium $)$ between 7-14 weeks of age. Athymic Nude mice 6-8 weeks old obtained from Harlan were inoculated s.c. with U87, glioblastoma astrocytoma $\left(2 \times 10^{6}\right.$ in $50 \mu \mathrm{l}$ medium). The mice were then used for in vivo studies when the tumors were $4-5 \mathrm{~mm}$.

In vivo PDT treatment: Prior to inoculation with tumor cells the whole right side of the BALB/c mice was shaved and depilated with Nair. This was not needed when Nudes were used. Upon reaching the appropriate treatment size $(4-5 \mathrm{~mm}$ diameter) the mice were injected i.v. via tail vain with the conjugates. 24 hours post injection the mice were restrained in Plexiglass holders without anesthesia, treated with a $1.1 \mathrm{~cm}$ diameter area of drug-activating laser light at $665 \mathrm{~nm}$ and a fluence of $128 \mathrm{~J} / \mathrm{cm}^{2}$ and a fluence rate of $14 \mathrm{~mW} / \mathrm{cm}^{2}$. The mice were observed daily after irradiation for tumor re-growth or tumor cure. Upon tumor recurrence measurements were taken using two orthogonal measurements Length and Width (perpendicular to L); volumes were calculated using the Microsoft Excel formula $\mathrm{V}=\mathrm{L}^{*} \mathrm{~W}^{2} / 2$ and recorded. Mice were considered cured if there were no palpable tumors by day 60; however, if the tumors reached $400 \mathrm{~mm}^{3}$ they were euthanized.

Tumor imaging: Three BALB/c mice per group bearing Colon 26 were imaged at three time points 24 , 48 and $72 \mathrm{~h}$ after being anesthetized with Ketamine/Xylazine, delivered intraperitonally or anesthetized with isofluorane. Compounds were imaged using a Maestro GNIR Flex In-vivo imaging system using a broadband excitation at $710-740 \mathrm{~nm}$ and an 800 nm long pass emission.

Tumor uptake (in vitro): In vitro cell uptake was determined by flow cytometry using an LSR II manufactured by Becton Dickson (BD). Colon 26 and U87 cells were seeded at $5.0 \times 10^{5}$ in 6 well plates in $2 \mathrm{ml}$ complete media for $24 \mathrm{~h}$. The conjugates were added at a concentration of $1 \mu \mathrm{M}$ and incubated in the dark at $37{ }^{\circ} \mathrm{C}$ for $24 \mathrm{~h}$. Cells in each well were harvested and placed in $5 \mathrm{ml}$ flow tubes with sieve caps, centrifuged cold at $4000 \mathrm{rpm}$ at $10{ }^{\circ} \mathrm{C}$ for 10 minutes. After removing the supernatant the cells were re-suspended in cold $300 \mu 12 \%$ FCS in PBS (FCM Buffer), placed on ice then ran on a BD LSR II. BD FACSDiva software was used to setup the parameters and acquire the data for the experiment. A variety of laser intercepts were used including the $405 \mathrm{~nm}$ Violet $(25 \mathrm{mw}$ Coherent Diode laser system), $488 \mathrm{~nm}$ Blue (20 mw primary laser, Coherent Solid State Laser system (Sapphire) and $640 \mathrm{~nm}$ Red (40 mw, Coherent "Cube" Solid State Laser system); however, for data analysis only those emission filter sets pertaining to the $405 \mathrm{~nm}$ Violet of $780 / 60,710 / 50$ and $660 / 20 \mathrm{~nm}$ were plotted using Microsoft Excel after the data was generated via FCS Express 4.0 as Microsoft PowerPoint slides.

\section{Results}

\section{Chemistry}

To investigate the impact of mono- vs. di- HPPH moieties in HPPH-CD conjugates, a series of indolenine- or benzindolenine cyanine dyes $1-5$, containing either an amino- or functionalities at various positions, were synthesized by following the methodology discussed in our preceding paper [12]. The HРPH 6, a photosensitizer (PS) selected for our study was either directly conjugated to CD $\mathbf{1}$ and $\mathbf{2}$ containing an amino-functionality or converted into the amino functionalized derivatives 7 and HPPH dimer 8 upon reacting with the respective amines (Figure 2). These $\mathrm{HPPH}$ analogs were isolated in modest yields (see the 
experimental section). Conjugate 9, a known bifunctional agent [6] and our lead compound was prepared to compare its imaging and therapeutic potential with other candidates including conjugate $\mathbf{1 0}$, which is very similar to 9, except the benzoindole moiety was replaced with the indole moiety; which should exhibit absorption shifted towards shorter wavelength resulting in an increased spectral overlap between the HPPH fluorescence and the cyanine dye absorption. Thus, in compound $\mathbf{1 0}$ stronger FRET should occur, resulting in lower singlet oxygen yield when compared to conjugate 9 (Figure 3 ).

For investigating the impact of mono- vs.
di-HPPH moieties in HPPH-CD conjugates $\mathbf{1 1}$ and $\mathbf{1 2}$ in which two HPPH moieties were linked with bezindolenine- or indolenine cyanine dyes 3 and 4 bearing a $m$-carboxylic phenylthiol group were prepared in $>60 \%$ yield.

In another approach, the amine functionalized HPPH 7 was reacted with cypate 5 bearing two carboxylic acid functionality following the well-established methodology used in peptide synthesis. This resulted in the isolation of the mono- and di- HPPH cypate conjugates $\mathbf{1 3}$ and $\mathbf{1 4}$ in $16.5 \%$ and $49 \%$ yields respectively (Figures 4 and 5).
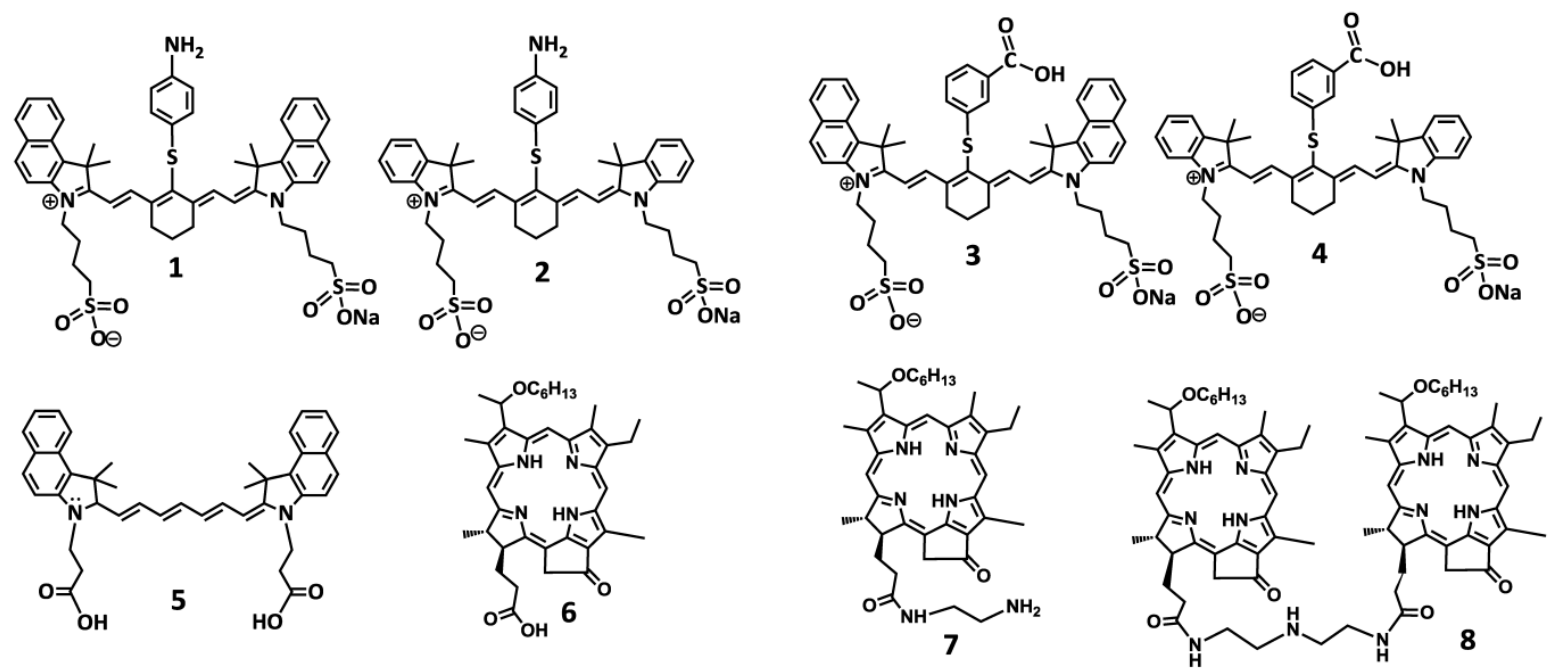

Fig 2. Structures of the cyanine dyes and photosensitizers used for the synthesis of bifunctional agents.

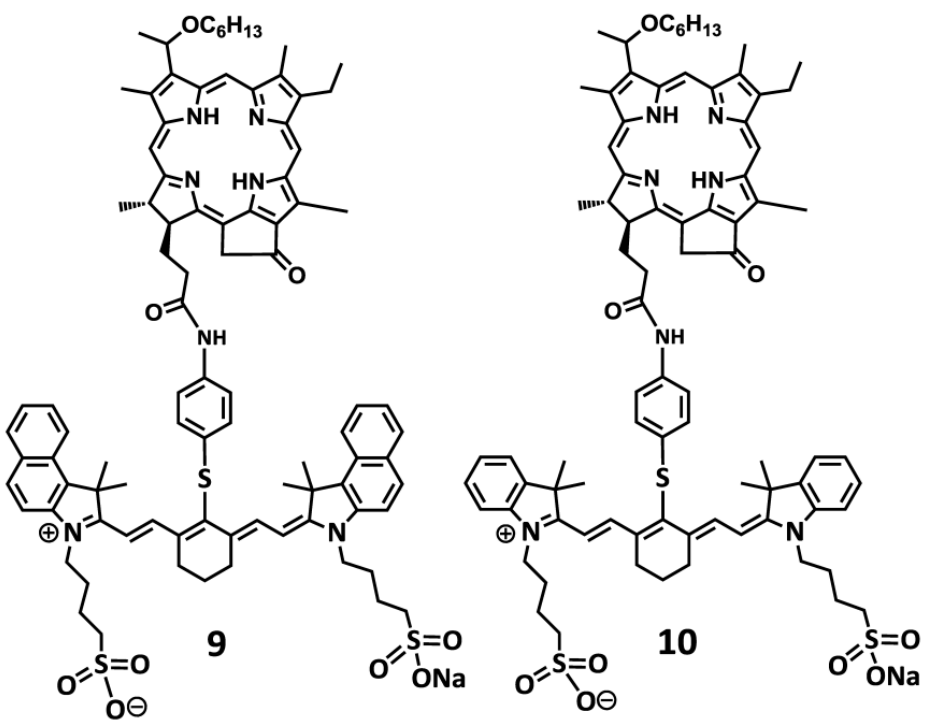

Fig 3. Structures of dual function agents in which the HPPH is conjugated to cyanine dyes containing bis-benzoindole and bis-indole moieties with amide linkages. 

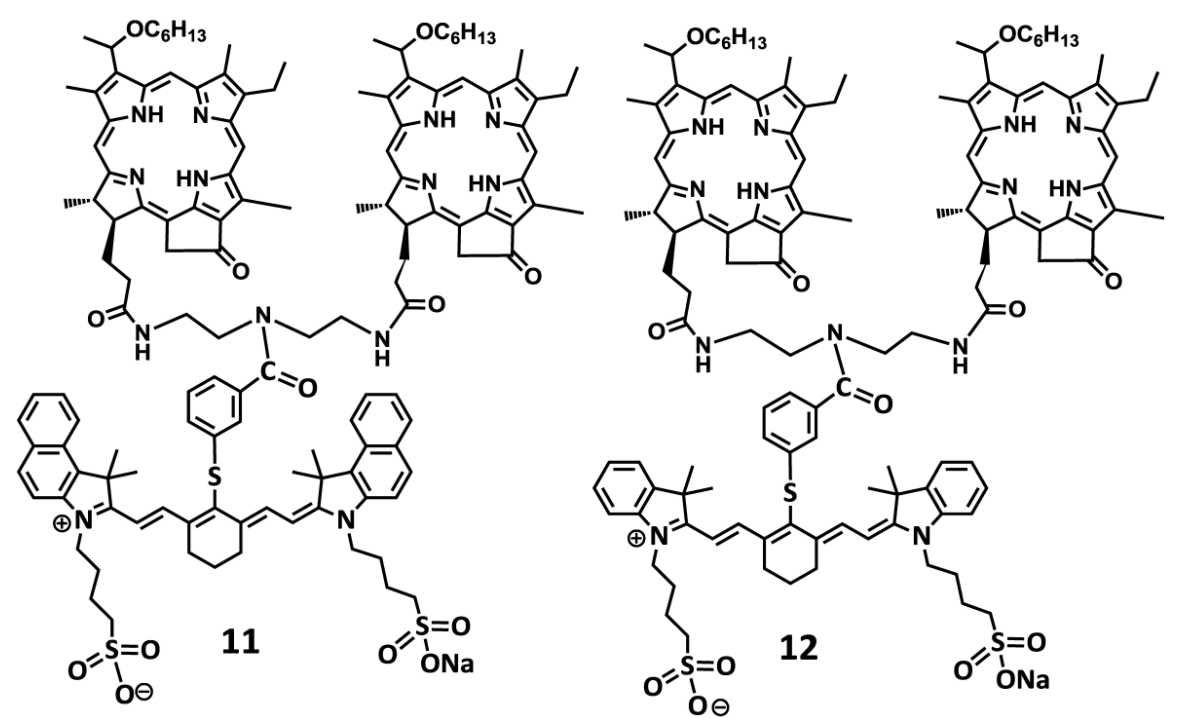

Fig 4. Structures of di-HPPH-bis-benzoindole and di-HPPH-bis-indole cyanine dyes joined with similar linkage.
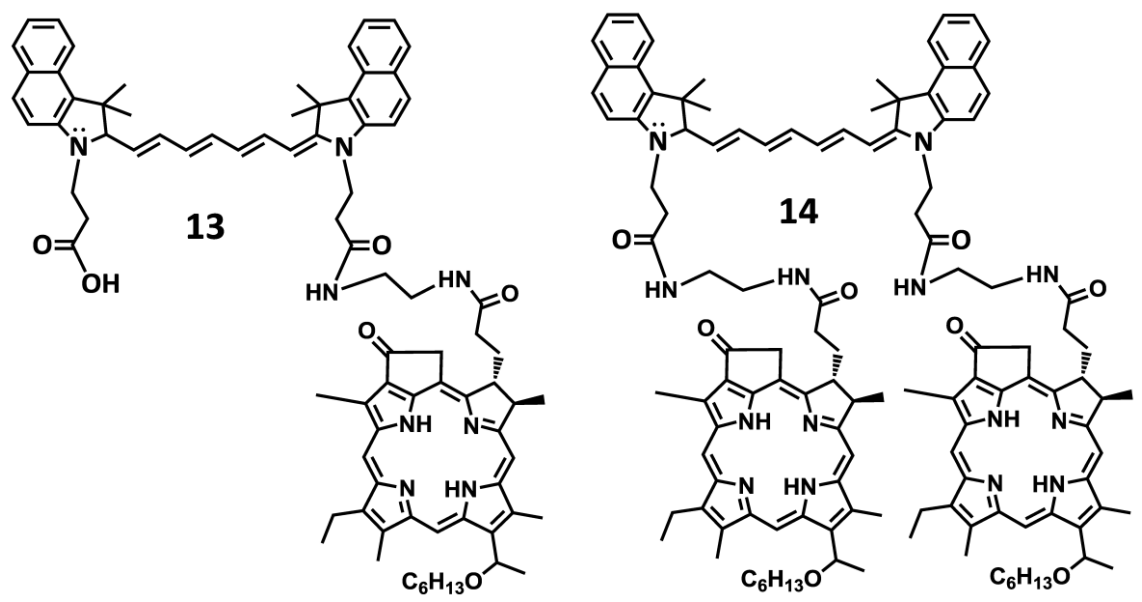

Fig 5. Structures of mono- and di-HPPH-cypate conjugate.

\section{Spectral Characterization}

The absorption and fluorescence spectra of HPPH, cyanine dyes 1, 5 and conjugates 9-14 are presented in Figure 6. Absorption spectra of conjugates demonstrate characteristic Soret and $\mathrm{Q}$ bands associated with the presence of the HPPH moiety (corresponding peaks are at $\sim 408$ and $\sim 660 \mathrm{~nm}$ ) as well as long-wave absorption by cyanine dye moieties. As suggested above, CD chromophores containing the benzindolenine moiety have absorption red-shifted in comparison with those containing indolenine moiety (corresponding peaks at $834-850 \mathrm{~nm}$ and 788-809 nm). Spectral position of CD absorption peak in the spectrum of $\mathbf{9}$ is close to that of $\mathbf{1}$, while $\mathrm{CD}$ absorption in $\mathbf{1 1}$ is little red-shifted in comparison with 1 and 9, suggesting that the availability of the second HPPH moiety in 11, influences the polarity of the surroundings for the CD chromophore and/or its conformation. Similar shift can be seen when comparing $\mathrm{CD}$ absorption by other conjugates, containing one and two HPPH chromophores, 10 and 12 (Figure 4, A). However, presence of second HPPH chromophore in $\mathbf{1 2}$ does not noticeably affect the fluorescence of CD moiety, as it does in $\mathbf{1 1}$ (Figure 4, B), suggesting that the benzindolenine moieties are more sensitive towards changes introduced by the second $\mathrm{HPPH}$ chromophore than the indolenine ones. It should be noted that the HPPH fluorescence in all conjugates is strongly quenched, due to strong electronic excitation energy transfer between $\mathrm{HPPH}$ and the CD chromophores [6]. The energy transfer in conjugate not only causes decrease in the HPPH moiety fluorescence, but also results in decreased efficiency in excitation of the triplet level of the HPPH moiety, which takes place via intersystem crossing, and corresponding reduction in singlet oxygen production This reduction is 
clearly seen in the results of the direct measurements of the singlet oxygen generated by the investigated compounds (Figure 7). As one can see, the singlet oxygen generation by the HPPH moiety in conjugates is strongly reduced when compared to that by the free, unconjugated HPPH. It should be noted that the addition of the second HPPH moiety in $\mathbf{1 1}$ and $\mathbf{1 4}$ increased singlet oxygen generation when compared to the corresponding conjugates containing single HPPH moiety, 9 and 13 (Figure 7). This effect is not, however, observed for the conjugates with CD chromophore containing indolenine moiety (10 and $\mathbf{1 2}$ in Figure 7), apparently because the energy transfer there is stronger, due to larger overlap between $\mathrm{HPPH}$ emission and $\mathrm{CD}$ absorption.
Table I: Longest wavelength absorbance, fluorescence peaks with Stokes shifts and comparative singlet oxygen yields of HPPH, cypate and the related HPPH-CD conjugates.

\begin{tabular}{|c|c|c|c|c|}
\hline Compounds & $\left(\lambda_{\max }\right)$ & $\left(\lambda_{\mathrm{em}}\right)$ & $\begin{array}{c}\text { Stokes } \\
\text { Shift }\end{array}$ & $\begin{array}{c}\text { Singlet Yield, } \\
\left({ }^{1} \mathrm{O}_{2}\right) \% \\
\end{array}$ \\
\hline HPPH & 660 & 666 & 6 & 45 \\
\hline CD (1) & 834 & 874 & 40 & 0 \\
\hline $\mathrm{CD}(2)$ & 795 & 829 & 34 & 0 \\
\hline$C D(3)$ & 832 & 866 & 34 & 0 \\
\hline$C D(4)$ & 795 & 829 & 34 & 0 \\
\hline CYPATE (5) & 785 & 822 & 37 & 0 \\
\hline HPPH-CD (9) & 834 & 874 & 40 & 8 \\
\hline HPPH-NH ${ }_{3}(10)$ & 796 & 831 & 35 & 5 \\
\hline $\mathrm{HPPH}_{2} \mathrm{CD}(11)$ & 850 & 890 & 40 & 9 \\
\hline $\mathrm{HPPH}_{2} \mathrm{NH}_{3}(12)$ & 809 & 842 & 33 & 6 \\
\hline НPPH-Сур (13) & 788 & 828 & 40 & 6 \\
\hline $\mathrm{HPPH}_{2} \mathrm{Cyp}$ (14) & 798 & 836 & 38 & 8 \\
\hline
\end{tabular}
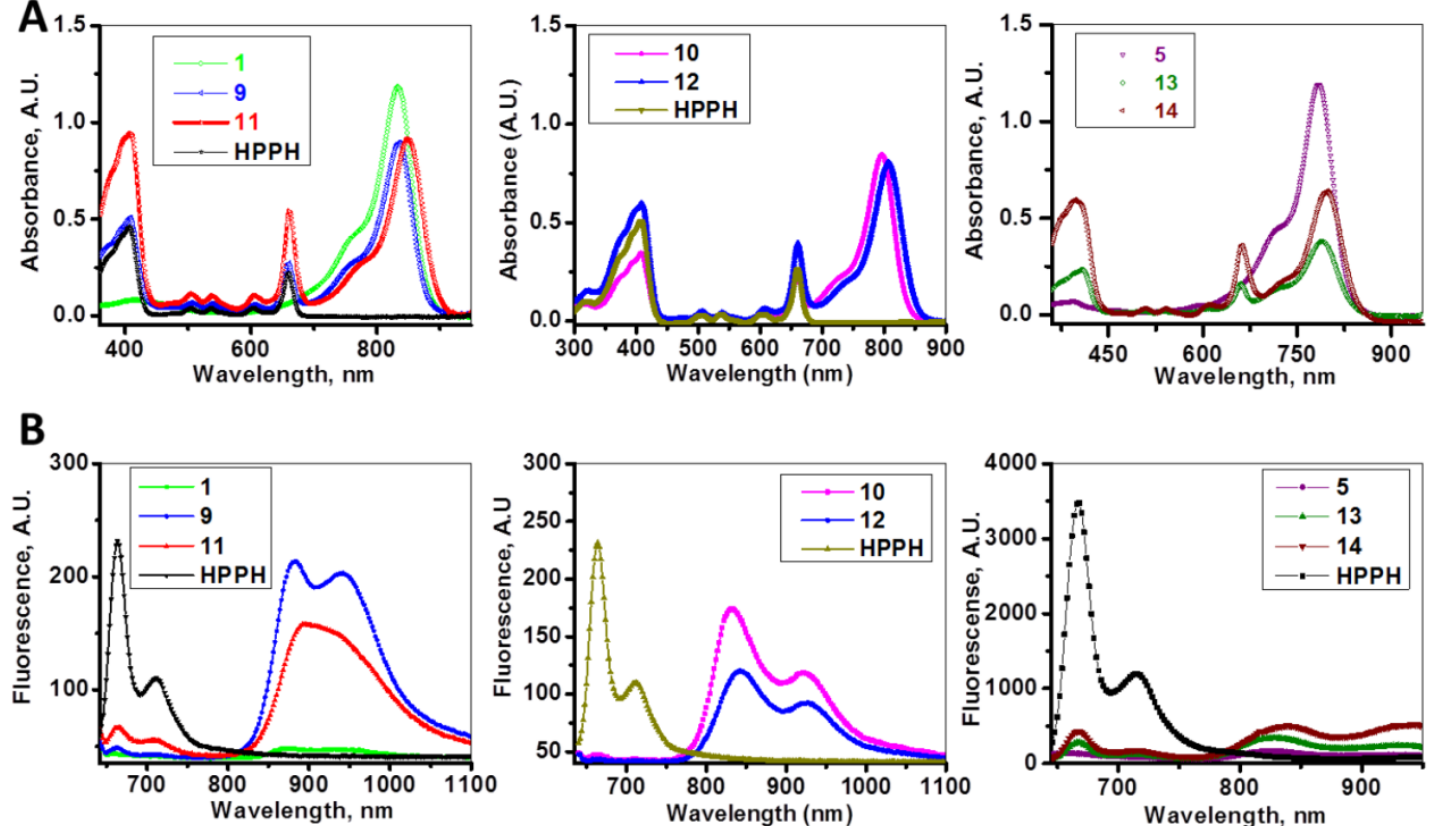

Fig 6. Absorption (A) and fluorescence (B) spectra of of HPPH, conjugates 9-14 and their respective fluorophores (I and 5).in). Solutions in methanol, $\mathrm{C}=5 \mu \mathrm{M}$.

A

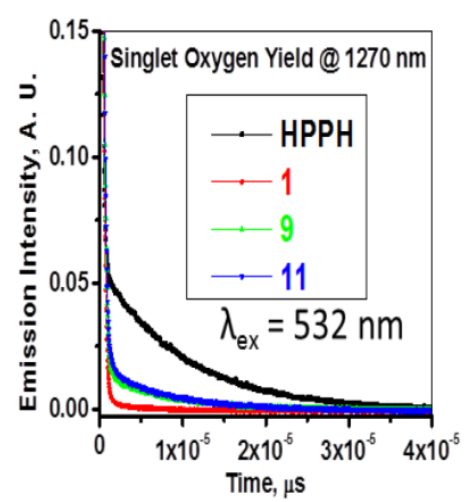

B

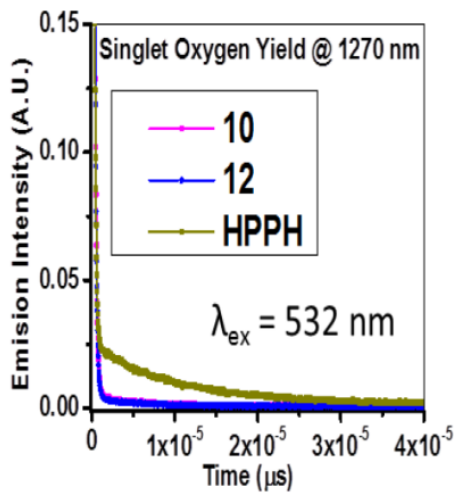

C

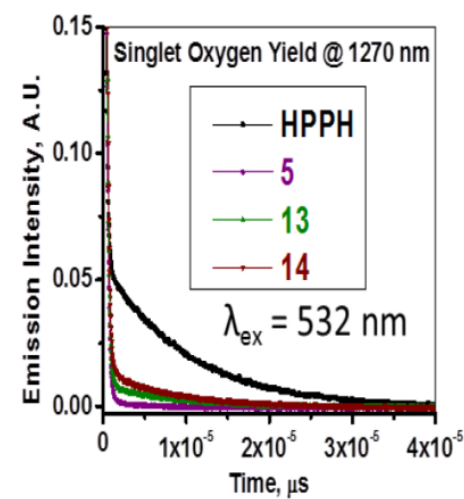

Fig 7. Time-resolved detection of singlet oxygen sensitized by HPPH, cyanine dyes I, 5 and conjugates $\mathbf{9 - 1 4}$. The recorded decays of the singlet oxygen phosphorescence at I $270 \mathrm{~nm}$ are shown. Absorbance of the irradiated samples (methanol solutions) was matched at the wavelength of excitation (532 nm). 
Comparative Biological Studies: In the initial study, HPPH-CD conjugates 9-14 were investigated for in vitro photosensitizing efficacy in Colon 26 (colon carcinoma) and U87 (glioblastoma) tumor models and in vivo efficacy was determined in BALB/c mice bearing Colon 26 tumors. After establishing a correlation between structural alterations and FRET [9], tumor uptake/specificity and PDT efficacy, the selected candidates were investigated for their imaging and therapeutic potential in U87 tumor model.

In vitro cell studies: In vitro cell uptake was determined by flow cytometry in Colon 26 and U87 cells using an LSR II manufactured by Becton Dickson (BD). Colon 26 and U87 cells were incubated in 6-well plates at $1 \times 10^{5}$ cells per well. After $48 \mathrm{~h}$ conjugates 9-14 were added and incubated for $24 \mathrm{~h}$ at a drug dose of $1 \mu \mathrm{M}$. Upon harvesting and filtering cells in cold 2 $\%$ FCS and Phosphate Buffer Saline (PBS) they were excited at $405 \mathrm{~nm}$.

A variety of laser intercepts were used including the $405 \mathrm{~nm}$ Violet $(25 \mathrm{mw}$ Coherent Diode laser system), $488 \mathrm{~nm}$ Blue (20 mw primary laser, Coherent Solid State Laser system (Sapphire) and $640 \mathrm{~nm}$ Red (40 mw, Coherent "Cube" Solid State Laser system); however, for data analysis only those emission filter sets pertaining to the $405 \mathrm{~nm}$ Violet of 780/60, 710/50 and $660 / 20 \mathrm{~nm}$ were plotted. From the results sum-

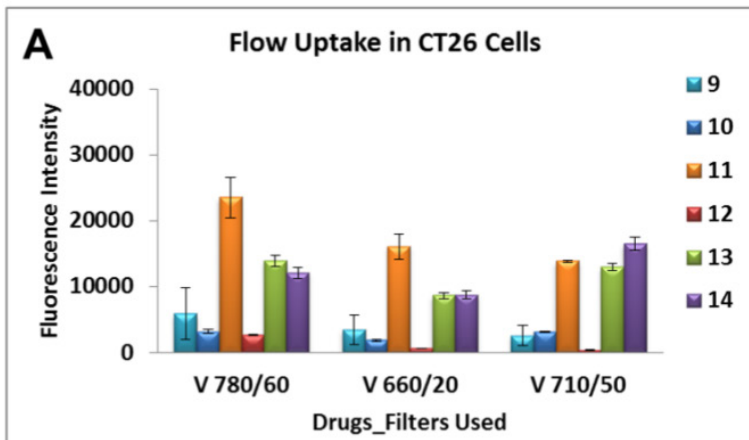

marized in Figure $\mathbf{8}$ it can be seen that conjugate $\mathbf{1 1}$ showed 5-10 fold higher uptake in both cell lines over 9, 10 and $\mathbf{1 2}$ when examined using the emission filter sets pertaining to the $405 \mathrm{~nm}$ Violet of $780 / 60,710 / 50$ and $660 / 20 \mathrm{~nm}$. Additionally, the mono- and di-HPPH cypates, $\mathbf{1 3}$ and $\mathbf{1 4}$ respectively, were also taken up in a higher quantity than $\mathbf{9 , 1 0}$ and $\mathbf{1 2}$ in both cell lines.

In vivo fluorescence imaging: $B A L B \backslash c$ mice bearing Colon 26 tumors were injected intravenously (i.v.) with conjugates 10-14 (dose: $0.03 \mu \mathrm{mol} / \mathrm{kg}$ ), and imaged at $24 \mathrm{~h}, 48 \mathrm{~h}$, and $72 \mathrm{~h}$ post injection (p.i) using a Maestro GNIR Flex In-vivo imaging system and a broadband excitation at max $710-740 \mathrm{~nm}$ and an 800 $\mathrm{nm}$ long pass emission. The conjugates showed significant tumor-affinity (Figure 9), although they were not excited at their optimal absorbance peak, due to the unavailability of the lasers at their respective absorbance maxima. However, based on the whole body pharmokinetic biodistribution of 10-14 at various time points the optimal time point for PDT treatment was determined to be $24 \mathrm{~h}$ because it was observed that there was no significant change in the uptake of the conjugates in the range of $24 \mathrm{~h}-96 \mathrm{~h}$ (see supplemental data). However, the in vivo biodistribution of one of the conjugates 11 is shown in Figure 10.

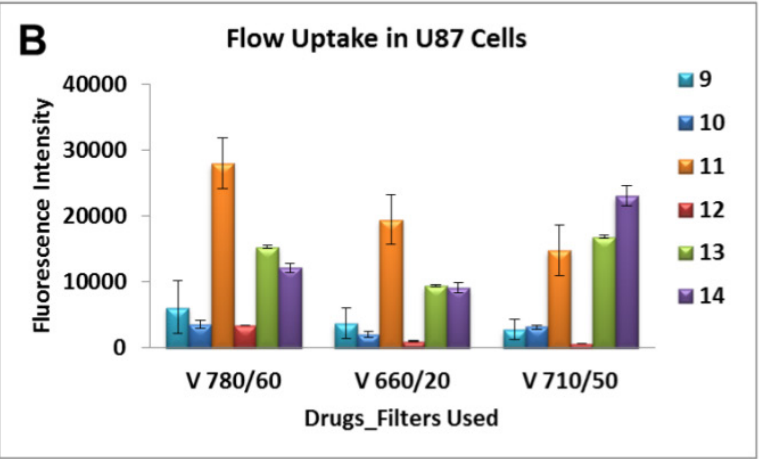

Fig 8. Characterization of the cellular uptake by the flow cytometry. Uptake of compounds 9 - I 4 were assessed at I $\mu$ M in Colon 26 and U87 tumor cells. A variety of laser intercepts were used including the $405 \mathrm{~nm}$ Violet ( $25 \mathrm{mw}$ Coherent Diode laser system), $488 \mathrm{~nm}$ Blue (20 mw primary laser, Coherent Solid State Laser system (Sapphire) and $640 \mathrm{~nm}$ Red (40 mw, Coherent "Cube" Solid State Laser system); however, for data analysis only those emission filter sets pertaining to the $405 \mathrm{~nm}$ Violet of $780 / 60,710 / 50$ and $660 / 20 \mathrm{~nm}$ were plotted.

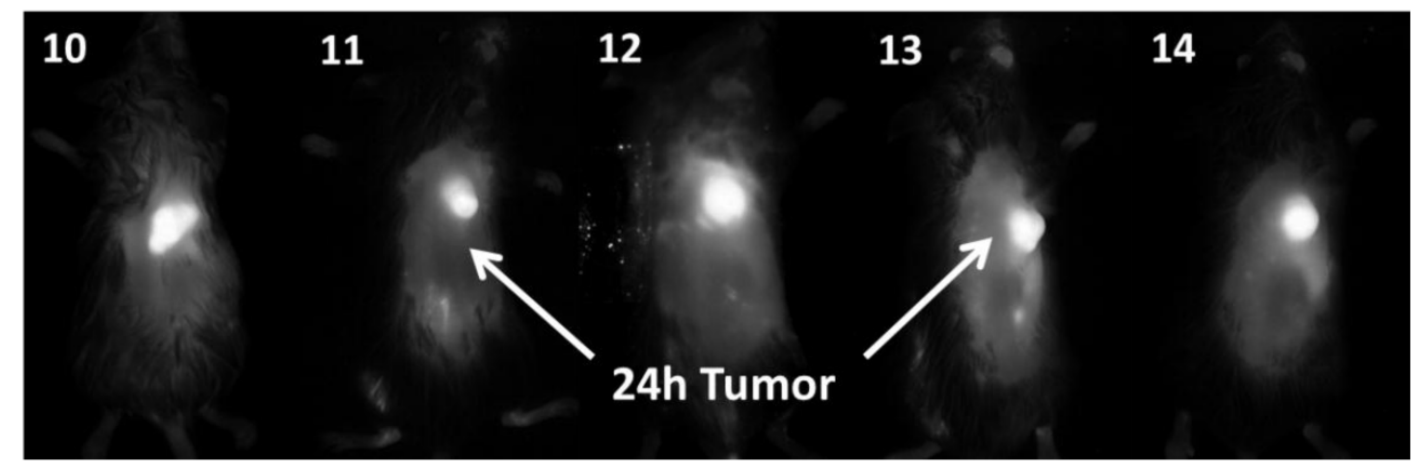

Fig 9. Whole body images of I 0, I I, I 2, I3 (H-Cyp) and I 4 ( $\mathrm{H}_{2}$-Cyp) at $24 \mathrm{~h}$ post-injection dose: $\left.0.03 \mu \mathrm{mol} / \mathrm{kg}\right)$. The images were taken using a Maestro GNIR Flex In-vivo imaging system using a broad and excitation at $710-740 \mathrm{~nm}$ and an $800 \mathrm{~nm}$ long pass emission. 


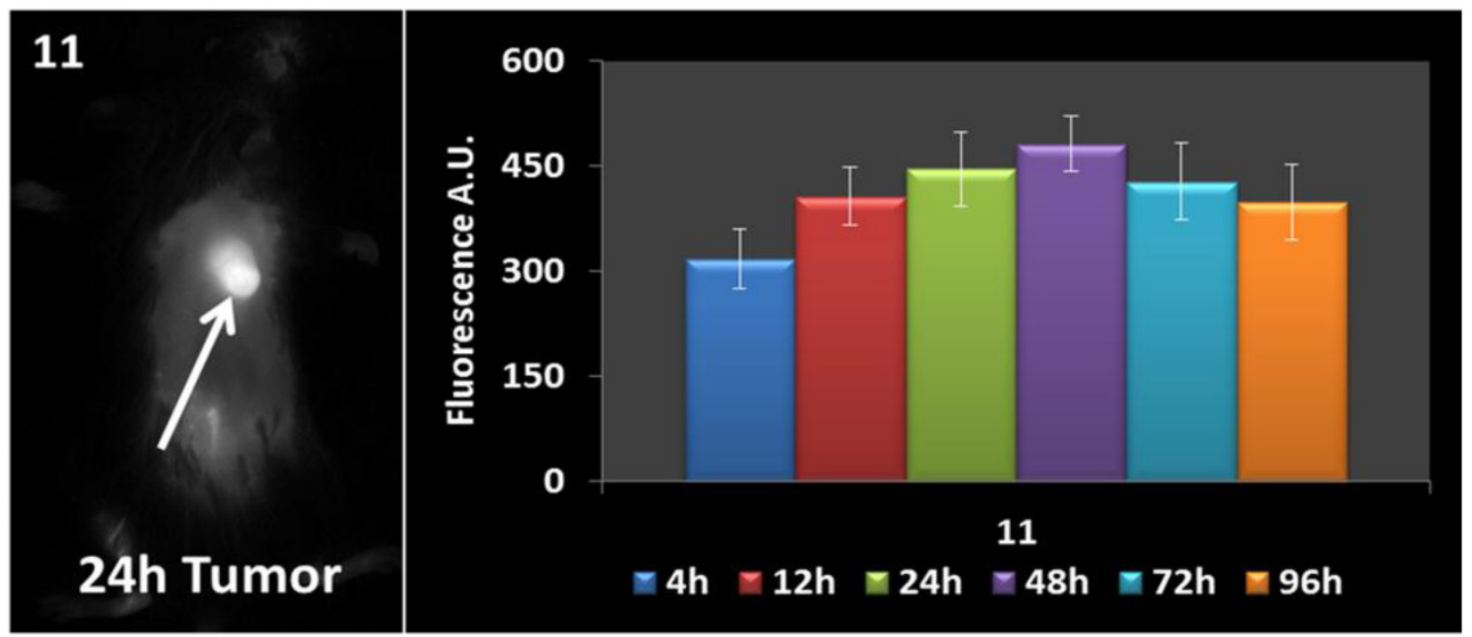

Fig 10. Whole body images of II at $24 \mathrm{~h}$ post-injection, and the fluorescence biodistribution at various time points. Due to the pharmacokinetic biodistribution, the optimal treatment time was determined to be $24 \mathrm{~h}$ because there was no significant change in the drug uptake beyond that point. The images were taken using a Maestro GNIR Flex In-vivo imaging system using a broadband excitation at 7/0 - 740 nm and an $800 \mathrm{~nm}$ long pass emission.

In vitro PDT Efficacy: Colon 26 cells were grown in RPMI-1640, whereas U87 cells were grown in MEM containing 10 \% Fetal Calf Serum (FCS), 1 \% L-glutamine, and Penicillin/Steptomycin (P/S). They were maintained at $5 \% \mathrm{CO}_{2}, 95 \%$ air and $100 \%$ humidity. Colon 26 and U87 cells were incubated in 96-well plates at various concentrations with conjugates 9, 10, 11 and 12 at $665 \mathrm{~nm}$. They were irradiated at variable light doses ranging from $0-4 \mathrm{~J} / \mathrm{cm}^{2}$ after $24 \mathrm{~h}$ incubation. Upon irradiation the plates were further incubated for $44 \mathrm{~h}$ at $37^{\circ} \mathrm{C}$ in the dark. Cells were later treated with $10 \mu \mathrm{lof} 4.0 \mathrm{mg} / \mathrm{ml}$ solution of MTT (3-[4,5-dimethylthiazol-2-yl]-2,5-diphenyltetraz olium bromide) dissolved in PBS. This was allowed to incubate for another $4 \mathrm{~h}$. After $4 \mathrm{~h}$ the MTT was removed and $100 \mu$ l of dimethyl sulfoxide was added to solubilize the formazan crystals. The MTT cell viability or phototoxicity assays were measured by reading the 96-well plate on a microtiter plate reader at an absorbance of $560 \mathrm{~nm}$. All compounds were formulated in $1 \%$ Tween-80/D5W for solubility in aqueous solution and diluted in complete medium for all in vitro studies. The results were plotted with the corresponding drug dose at a light dose of $4 \mathrm{~J} / \mathrm{cm}^{2}$ using the programs Microsoft Excel and Origin 8.6, Figure 11. The $\mathrm{EC}_{50}$ doses were calculated to compare the efficacy of the compounds. As can be seen, in both cell lines conjugate $\mathbf{1 1}$ appeared to be the most effective and had the lowest $\mathrm{EC}_{50}$ among all the conjugates, Table 2. Interestingly, conjugate 12 in which the bisbenzoindole $\mathrm{CD}$ was replaced by bis-indole $\mathrm{CD}$ was the least cytotoxic conjugate when compared to the other bifunctional agents $(\mathbf{9}, \mathbf{1 0}$ and 11) in Colon 26, but was similar to $\mathbf{1 1}$ in U87 cells.
Table 2. Comparative $\mathrm{EC}_{50}$ values (50\% cell kill) of compounds $\mathbf{9}$, I $\mathbf{0}, \mathbf{I}$ I and I $\mathbf{2}$ in Colon 26 and U87 tumor models.

\begin{tabular}{lll}
\hline Compounds & EC $_{50}$ of Colon $26(\mu \mathrm{M})$ & EC $_{50}$ of $U 87(\mu \mathrm{M})$ \\
\hline 9 & 0.25 & 1.63 \\
10 & 0.26 & 0.94 \\
11 & 0.13 & 0.64 \\
12 & 0.70 & 0.66 \\
\hline
\end{tabular}

Comparative in vivo PDT Efficacy: For determining preliminary in vivo efficacy, five BALB/c mice / group, 5 - 8 weeks of age were inoculated subcutaneously (s.c.) on the right posterior shoulder with Colon 26 ( $1 \times 10^{6}$ cells in $50 \mu \mathrm{l}$ medium) between 7 - 14 weeks of age. Prior to inoculation with tumor cells the whole right side of the mice were shaved and depilated with Nair, Figure 12. Additionally, athymic Nude mice were inoculated s.c. on the right posterior shoulder with U87 cells ( $2 \times 10^{6}$ cells in $50 \mu$ l medium). Upon reaching the appropriate treatment size (4-5 $\mathrm{mm}$ diameter) of tumors, the mice were injected intravenously (i.v.) via tail vain. Initially studies were performed with conjugates $\mathbf{9}$ and $\mathbf{1 1}$ at variable drug doses $3.5,2.5,1.5,0.75$ and $0.4 \mu \mathrm{mol} / \mathrm{kg}$, in order to find the optimal drug dose. Once the optimal drug dose was identified as $1.5 \mu \mathrm{mol} / \mathrm{kg}$, candidates $\mathbf{1 0}$ and 12 were also assessed for their PDT efficacy. Conjugates 9 - 12 were further accessed using a larger group (10 Nude mice). At $24 \mathrm{~h}$ post injection the mice were restrained in Plexiglass holders without anesthesia and treated with a $1.1 \mathrm{~cm}$ diameter area of drug-activating laser light at $665 \mathrm{~nm}$ using a low and slow total light dose of $128 \mathrm{~J} / \mathrm{cm}^{2}$ and a fluence rate of $14 \mathrm{~mW} / \mathrm{cm}^{2}$ (Figure 13), a combined fluence and flu- 
ence rate that was previously found to be very effective in terms of PDT response $[13,14]$.

The mice were observed daily after irradiation for tumor re-growth or tumor cure. Upon tumor recurrence measurements were taken using two orthogonal measurements Length and Width (perpendicular to L); volumes were calculated using the Microsoft Excel formula $\mathrm{V}=\mathrm{L}^{*} \mathrm{~W}^{2} / 2$ and recorded. Mice were considered cured if there were no palpable tumors by day 60; however, if the tumors reached 400 $\mathrm{mm}^{3}$ they were euthanized and Kaplan Meier Survival curves were drawn to depict PDT response.

From the data summarized in Figure 13, it can be seen that conjugate 9 (HPPH-CD, the lead compound) at a dose of 3.5 and $2.5 \mu \mathrm{mol} / \mathrm{kg}$, gave $40 \%$ and $20 \%$ tumor response. Under similar treatment parameters, conjugate 11 showed $40 \%$ and $60 \%$ tumor cure respectively. Treatment of BALB/c mice bearing Colon 26 tumors with compounds $\mathbf{9 ,} \mathbf{1 0}, \mathbf{1 1}$ and $\mathbf{1 2}$ at 1.5 $\mu \mathrm{mol} / \mathrm{kg}$ drug dose, showed no long-term tumor cure with conjugate 9, whereas $40 \%, 80 \%$ and $20 \%$ cures were observed with conjugates 10, 11 and 12 respectively, Figure 13 and Table 3. Under similar treatment conditions, no tumor cure was observed with monoand di-HPPH cypate. In nude mice bearing U87 tumors, tumor cures were as follows: Conjugate 9: 30\% (3/10 mice were tumor free on day 60$)$; Conjugate 10: $40 \%$ response $(4 / 10$ were tumor-free on day 60$)$; Conjugate 11: $30 \%$ (3/10 were tumor free on day 60$)$, and conjugate $\mathbf{1 2}$ did not produce any cure, Table 3 .

Table 3. Summary of the in vitro MTT and flow uptake of 9,10, I I and I 2 in both Colon 26 and U87 are shown in the table above. Additionally, the corresponding in vivo tumor responses of $5 \mathrm{BALB} / \mathrm{c}$ mice inoculated with Colon 26 and 10 Nude mice inoculated with U87 and treated at the optimized dose of $1.5 \mu \mathrm{mol} / \mathrm{kg}$ are also shown.

\begin{tabular}{|c|c|c|c|c|c|c|c|c|}
\hline \multirow{3}{*}{ Compounds } & \multirow{3}{*}{$\begin{array}{c}\text { MTT } \\
\text { Light Dose } \\
\left(\mathrm{J} / \mathrm{cm}^{2}\right)\end{array}$} & \multicolumn{2}{|c|}{$\mathrm{EC}_{50}(\mu \mathrm{M})$} & \multicolumn{2}{|c|}{ Flow Uptake } & \multicolumn{3}{|c|}{ In Vivo PDT Efficacy } \\
\hline & & \multirow{2}{*}{ СТ 26} & \multirow{2}{*}{ U87 } & \multirow{2}{*}{ Ст 26} & \multirow{2}{*}{ U87 } & \multirow{2}{*}{$\begin{array}{l}\text { Drug Dose } \\
\text { ( } \mu \mathrm{mol} / \mathrm{kg})\end{array}$} & \multicolumn{2}{|c|}{ \% Tumor Cure } \\
\hline & & & & & & & СТ 26 & U87 \\
\hline $\begin{array}{c}9 \\
\text { (HPPH-CD) }\end{array}$ & 4 & 0.25 & 1.63 & 5911 & 6133 & 1.5 & 0 & 30 \\
\hline $\begin{array}{c}10 \\
\text { (HPPH-NH-3) }\end{array}$ & 4 & 0.26 & 0.94 & 3194 & 3485 & 1.5 & 40 & 40 \\
\hline $\begin{array}{c}11 \\
\left(\mathrm{HPPH}_{2} \mathrm{CD}\right)\end{array}$ & 4 & 0.13 & 0.64 & 23555 & 27980 & 1.5 & 80 & 30 \\
\hline $\begin{array}{c}12 \\
\left(\mathrm{HPPH}_{2}-\mathrm{NH}-3\right)\end{array}$ & 4 & 0.70 & 0.66 & 2698 & 3492 & 1.5 & 20 & 0 \\
\hline
\end{tabular}
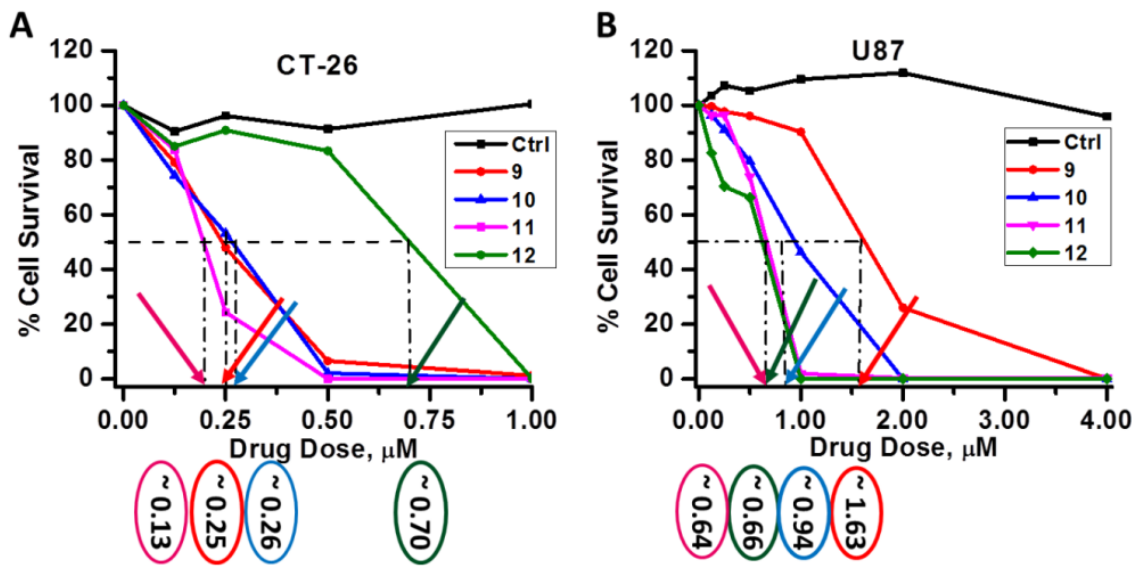

Fig II. MTT phototoxicity assays of $9-12$ were assessed in Colon 26 (a), and U87 (b) tumor cell lines at $4 \mathrm{~J} / \mathrm{cm}^{2} 48 \mathrm{~h}$ post incubation in the dark at $37{ }^{\circ} \mathrm{C}$ and measured by reading the 96 -well plate on a microtiter plate reader at an absorbance of $560 \mathrm{~nm}$. 


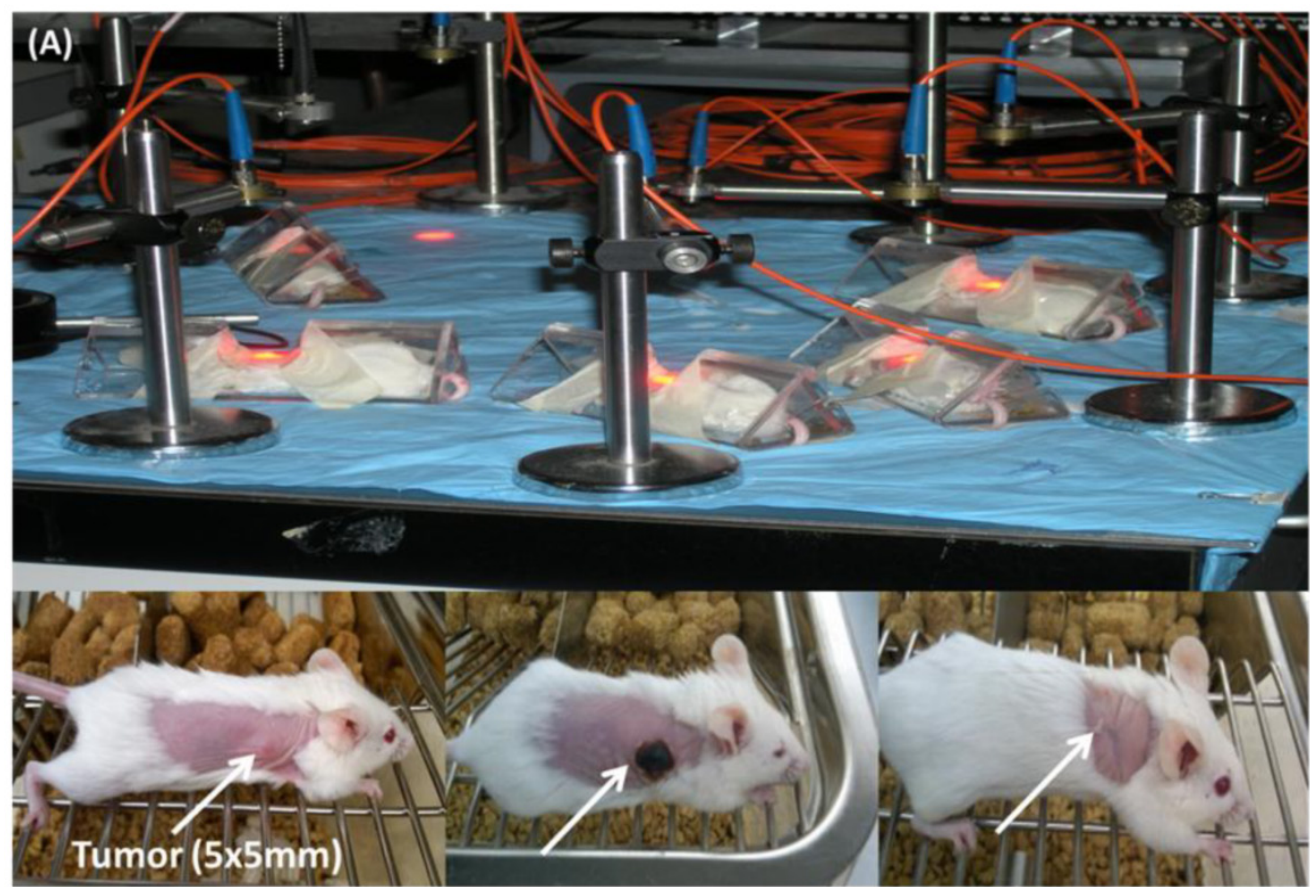

$\begin{array}{lll}\text { (B) BALB } \backslash c \text { mouse before PDT } & \text { (C) 7days after PDT }\end{array}$

(D) 30 to 60 days after PDT

Fig I 2. In vivo photosensitizing of conjugates in BALB/c mice bearing Colon 26 tumors having reached the appropriate treatment size of 4-5 mm diameter, at variable drug doses. The tumors were exposed to treatment conditions of $128 \mathrm{~J} / \mathrm{cm}^{2}$ and $14 \mathrm{~mW} / \mathrm{cm}^{2}$ with a 1 .I cm diameter of drug-activating laser light $(665 \mathrm{~nm})$ at $24 \mathrm{~h}$ post injection. Tumor regrowth or delay was observed 60 days or higher after treatment. If the treated area remained flat at day 60 or higher, this was considered cured if the tumors regrew to a volume of $400 \mathrm{~mm}^{3}$ the mice were euthanized.
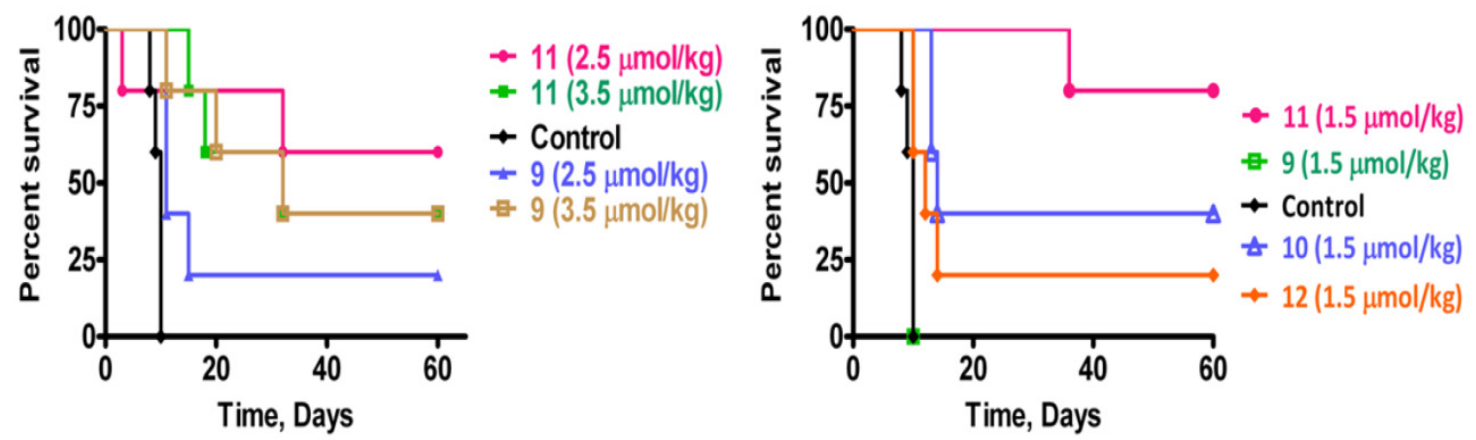

Fig 13. The PDT efficacy of 9 and II were assessed at 3.5 and $2.5 \mu \mathrm{mol} / \mathrm{kg}$ (A) and $\mathbf{9}, \mathbf{1 0}, \mathbf{I}$ I and I 2 were assessed at I.5 $\mu \mathrm{mol} / \mathrm{kg}$ (B). Tumors were exposed to light $\left(128 \mathrm{~J} / \mathrm{cm}^{2}, 14 \mathrm{~mW} / \mathrm{cm}^{2}\right)$ at $24 \mathrm{~h}$ post-injection. All in vivo studies were performed in BALB/c mice bearing Colon 26 tumors.

\section{Discussion}

At low and slow light treatment parameters, compared to mono-HPPH-benzindole $\mathrm{CD}$ 9, the di-HPPH-benzindole-CD (11) showed enhanced PDT efficacy. Among these two conjugates a direct correlation between tumor-uptake, singlet oxygen production and PDT efficacy was observed. These results demonstrate that increasing the number of $\mathrm{HPPH}$ moieties from one to two, enhances in vivo and in vitro PDT efficacy. During our studies we tested a series of compounds and it was evident from the in vivo PDT data that conjugate 11, Fig. 12, as well as some that are not yet published (manuscript) were less effective at higher doses of $3.5 \mu \mathrm{mol} / \mathrm{kg}$ and $2.5 \mu \mathrm{mol} / \mathrm{kg}$ than at the lower dose of $1.5 \mu \mathrm{mol} / \mathrm{kg}$. We attributed this occurrence to the complex conformational dynamics in longer arrays [15] which may result in aggregation of the conjugates causing the generation of less singlet oxygen.

It was also evident that the uptake of di-HPPH-bis-benzindole CD conjugate (11) increased approximately 5-10 fold in Colon 26 and U87 models compared to HPPH-benzindole-CD (9) and HPPHindole-CD (10). However, similar to conjugate 11, compounds 13 and 14 were also preferentially taken up by the colon 26 and U87 cells in vitro. This could possibly be explained by several theories [16]: 1) an 
acidic tumor can cause preferential accumulation of drugs that are prone to protonate and become more lipophilic as they traverse the tumors; 2) tumors contain macrophages that are able to ingest and monomerize photosensitizer aggregates and release lipoprotein bound drugs; 3 ) tumor surfaces tend to have more low-density lipoprotein (LDL) receptors than normal cells and lipophilic photosensitizers binds preferentially to lipoprotein; and 4) tumors tend to have a poor lymphatic drainage or leaky vasculature. Although, 10 was synthesized by conjugating HPPH with modified CD 2 (whose parent, IR783, showed better tumor uptake than other cyanine dyes, see our accompanying article in this journal) [12], its in vitro efficacy in Colon 26 cells was similar to 9; however, it was more efficacious than 9 in U87 in vitro. The in vivo studies of $\mathbf{1 0}$ in Colon 26 and Nude mice were also more efficacious than 9. This suggested that further modification of $\mathbf{1 0}$ by adding one more HPPH moiety as $\mathbf{1 1}$ should result in an even more pronounced effect in U87 tumors, especially since conjugates 9, 10 and 11, in general, accumulated more in U87 than Colon 26 cells as determined by flow cytometry assays. However, the in vivo results were significantly different. Modification of $\mathbf{1 0}$ with two HPPH moieties yielded compound 12 which did not show improved in vivo PDT in both BALB/c (Colon 26 cells) or Nudes (U87 cells). Conjugates $\mathbf{1 3}$ and $\mathbf{1 4}$ were inefficient PDT agents because they showed no cures in vivo. One explanation for the poor PDT response of $\mathbf{1 3}$ and $\mathbf{1 4}$ could be due to the photobleaching of the unstable polymethine chain on the cypate portion of the conjugate, by the singlet oxygen generated; leading to quick and complete degradation of the compounds upon laser light activation [16]. The disparity in in vitro and in vivo uptake, PDT efficacy among the conjugates could be due to a significant difference in pharmacokinetics of the conjugates. Other possible reason could be due to their rate of binding to the cellular membranes as well as the relationship between diffusion and endocytosis in each conjugate [17]. Also, PDT systemically ablates tumor cells by (a) singlet oxygen generation, (b) vascular shutdown and (c) invoking an immune response. Compromise of any of these routes could cause reduced PDT response. The variation in activity of the above mentioned conjugates could possibly be explained by conducting studies to probe their cell death mechanisms, which are currently underway and will be published separately.

\section{Conclusion}

In summary, the newly synthesized conjugates were compared with our lead compound HPPH-CD in terms of photophysical properties, in vitro and in vivo PDT efficacy, tumor uptake and imaging potential. All the HPPH-cyanine dye conjugates showed tumor-imaging capability even at a very low dose. However, conjugates 10, 12, 13 and 14 were visibly more pronounced, possibly due to the fact that their maximum absorbance are within proximity to the NIR excitation filter sets available at our imaging facility (broad band excitation of $710-740 \mathrm{~nm}$ with a $800 \mathrm{~nm}$ LP emission filter). Among the analogs investigated, the bifunctional agent in which two HPPH moieties were linked to the benzoindole-based cyanine dye (IR820), 11, produced enhanced tumor uptake and tumor contrast in both Colon 26 (a murine Colon carcinoma) and U87 (a human glioblastoma) cell lines. Compound $\mathbf{1 1}$ showed superiority over all the conjugates, especially, the lead compound (mono HPPH-cyanine dye 9). The long-term PDT efficacy of conjugate 11 in BALB/c mice, bearing Colon 26 tumors was enhanced; however, its efficacy in Nude mice bearing U87 tumors was intermediate.

\section{Supplementary Materials}

Ex vivo fluorescence biodistribution of conjugates 10, 13 and 14 at various time points, and the ${ }^{1} \mathrm{H}$ NMR spectra HPPH analogs (monomers and the corresponding cyanine dye conjugates).

http://www.thno.org/v03p0703s1.pdf

\section{Abbreviations}

NIRF: near infrared fluorophore; PDT: Photodynamic therapy; CD: Cyanine dye; FRET: Forster Resonance Energy Transfer; PS-NIRF: PS-near infrared fluorophore; RIF: radiation-induced fibrosarcoma; Colon 26: a murine Colon carcinoma cell line; U87: a human glioblastoma cell line.

\section{Acknowledgment}

The authors are highly thankful to $\mathrm{NIH}$ for financial support (NIH: RO1CA127369), research supplement to promote diversity in health-related research to Nadine S. James (RO1 CA 127369S) and program project grant (PO1 CA55791). Mass spectrometry analyses of the compounds were performed at the Michigan State University, East Lansing, Michigan and the University at Buffalo, Buffalo, NY, 14263.

\section{Conflict of Interest}

The authors have declared that no conflict of interest exists.

\section{References}

1. Henderson BW, Dougherty TJ. How does photodynamic therapy work? Photochemistry and Photobiology. 1992; 55: 145-57. 
2. Konan YN, Gurny R, Allemann E. State of the art in the delivery of photosensitizers for photodynamic therapy. J Photochem Photobiol, B. 2002; 66: 89-106.

3. Prasad PN. Introduction to Biophotonics. Hoboken, USA: John Wiley \& Sons, Inc; 2003.

4. Kim S, Ohulchanskyy TY, Pudavar HE, Pandey RK, Prasad PN. Organically Modified Silica Nanoparticles Co-encapsulating Photosensitizing Drug and Aggregation-Enhanced Two-Photon Absorbing Fluorescent Dye Aggregates for Two-Photon Photodynamic Therapy. Journal of the American Chemical Society. 2007; 129: 2669-75.

5. Yan F, Kopelman R. The embedding of meta-tetra(hydroxyphenyl)-chlorin into silica nanoparticle platforms for photodynamic therapy and their singlet oxygen production and ph-dependent optical properties. Photochemistry and Photobiology. 2003; 78: 587-91.

6. Chen Y, Gryshuk A, Achilefu S, Ohulchansky T, Potter W, Zhong T, et al. A Novel Approach to a Bifunctional Photosensitizer for Tumor Imaging and Phototherapy. Bioconjugate Chemistry. 2005; 16: 1264-74.

7. Chen Y, Ohkubo K, Zhang M, Wenbo E, Liu W, Pandey Suresh K, et al. Photophysical, electrochemical characteristics and cross-linking of STAT-3 protein by an efficient bifunctional agent for fluorescence image-guided photodynamic therapy. Photochem Photobiol Sci. 2007; 6: 1257-67.

8. Srivatsan A, Ethirajan M, Pandey SK, Dubey S, Zheng X, Liu T-H, et al. Conjugation of cRGD Peptide to Chlorophyll a Based Photosensitizer (HPPH) Alters Its Pharmacokinetics with Enhanced Tumor-Imaging and Photosensitizing (PDT) Efficacy. Mol Pharmaceutics. 2011; 8: 1186-97. doi:10.1021/mp200018y.

9. Lovell JF, Chen J, Jarvi MT, Cao W-G, Allen AD, Liu Y, et al. FRET Quenching of Photosensitizer Singlet Oxygen Generation. Journal of Physical Chemistry B. 2009; 113: 3203-11.

10. Pandey RK, Bellnier DA, Smith KM, Dougherty TJ. Chlorin and porphyrin derivatives as potential photosensitizers in photodynamic therapy. Photochemistry and Photobiology. 1991; 53: 65-72.

11. Ye Y, Bloch S, Kao J, Achilefu S. Multivalent Carbocyanine Molecular Probes: Synthesis and Applications. Bioconjugate Chemistry. 2005; 16: 51-61.

12. James NS, Chen $Y$, Joshi P, Ohulchanskyy TY, Ethirajan M, Henary M, Strekowski L, Pandey RK. Evaluation of Polymethine Dyes as Potential Probes for Near Infrared Fluorescence Imaging of Tumors: Part 1. Theranostics 2013; 3(9):692-702.

13. Snyder JW, Greco WR, Bellnier DA, Vaughan L, Henderson BW. Photodynamic Therapy: A Means to Enhanced Drug Delivery to Tumors. Cancer Research. 2003; 63: 8126-31.

14. Henderson BW, Busch TM, Snyder JW. Fluence rate as a modulator of PDT mechanisms. Lasers in surgery and medicine. 2006; 38: 489-93.

15. Kuimova MK, Balaz M, Anderson HL, Ogilby PR. Intramolecular rotation in a porphyrin dimer controls singlet oxygen production. Journal of the American Chemical Society. 2009; 131: 7948-9. doi:10.1021/ja901237s.

16. Juzeniene A, Peng $Q$, Moan J. Milestones in the development of photodynamic therapy and fluorescence diagnosis. Photochemical \& Photobiological Sciences. 2007; 6: 1234-45.

17. Minnes R, Weitman H, You Y, Detty Michael R, Ehrenberg B. Dithiaporphyrin derivatives as photosensitizers in membranes and cells. The journal of physical chemistry B. 2008; 112: 3268-76. 